\title{
One-rod core microstructured optical fibers for evanescent sensing applications: an alternative approach
}

Dinesh Kumar Sharma ( $\nabla$ dk81.dineshkumar@gmail.com )

Indian Institute of Technology Kanpur https://orcid.org/0000-0001-5461-5199

Saurabh Mani Tripathi

IIT Kanpur: Indian Institute of Technology Kanpur

\section{Research Article}

Keywords: Microstructured optical fiber, Polymethylmethacrylate, Evanescent field, Sensitivity coefficient, Confinement loss, Overlapping factor

Posted Date: April 26th, 2021

DOI: https://doi.org/10.21203/rs.3.rs-339315/v1

License: (c) (i) This work is licensed under a Creative Commons Attribution 4.0 International License.

Read Full License 


\title{
One-rod core microstructured optical fibers for evanescent sensing applications: an altrernative approach
}

\author{
Dinesh Kumar Sharma ${ }^{1, *}$ and Saurabh Mani Tripathi ${ }^{1,2}$ \\ ${ }^{1}$ Center for Lasers and Photonics, Indian Institute of Technology Kanpur, Kanpur-208016, \\ India \\ ${ }^{1,2}$ Department of Physics, Indian Institute of Technology Kanpur, Kanpur-208016, India \\ dineshk@iitk.ac.in*,smt@iitk.ac.in
}

\begin{abstract}
The advent of polymer (or plastic) optical fibers (POFs) with porous cross-section has contributed significantly to the sensing applications. We intend to explore the sensing attributes of polymer-based one-rod core microstructured optical fibers (P-MOFs) via utilizing an auxiliary approach. The sensitivity coefficient and the overlapping factor of PMOF's introduced design in air-filled and water-filled layouts against the structural parameters are exploited. We reported the overlapping factor of $\sim 94 \%$ and $\sim 82 \%$ for the said configurations of the fibers, respectively, at the wavelength of $0.633 \mu \mathrm{m}$. The confinement loss, mode-index, and effective mode-area are also evaluated for the anticipated structures. For assessing the confinement loss, an alternative approach based on double-clad fiber (DCF) approximation is employed. The validity of the simulated results is scrutinized with those as documented in the literature. Relative errors are also tabulated.
\end{abstract}

Keywords: Microstructured optical fiber; Polymethylmethacrylate; Evanescent field; Sensitivity coefficient; Confinement loss; Overlapping factor.

\section{Introduction}

The need for low-cost and easy-to-use sensing systems for the rapid screening of contaminants is constantly increasing. Traditional monitoring techniques are typically based on laboratory analyses of representative field-collected samples; this necessitates considerable time, effort, expense, and the sample composition may change before examination [1-3]. Alternatively, portable monitoring systems relying on sensing methods appear well suited to complement standard analytical techniques. They can also be permanently installed at the monitoring sites and can transmit the data remotely. Bio and chemo receptor-based sensors in polymer-based optical fibers [4-6] are well suited for 
numerous applications. Polymer (or plastic) optical fibers (POFs) possess predominant supremacy over their glass counterparts and assist as an accessory in the communication network. The miscellaneous applications accompanied by POFs are explored and have been designed for financial gain. Polymer materials possessed additional advantages such as high sensitivity to strain, high fracture rough handling, and feasible negative thermo-optic coefficients. Furthermore, they have excellent concordance with organic materials facilitating their strong candidature for many applications such as in biomedical [1-7]. POFs are comparatively cheaper and flexible; moreover, they are convenient to cut and splice, making them an exemplary contestant for the local area network. Additionally, they are proportionately easy to handle during the preparation, supported by their sizeable air-hole diameter. Compared to silica fibers, POFs certainly possessed tremendous importance, such as a lower Young's modulus, a higher thermo-optic coefficient, and a more significant elongation before breakage, exploited in a large variety of applications [8-10]. They can be employed in advanced strain circumstances where silica-based fibers may lapse; moreover, their large core dimension minimizes core alignment requirement during connection [11-13].

The development of microstructured optical fibers (MOFs) [14-17] confers the perspective of improved sensing without etching the coating and cladding, and thus, preserving the compactness of the sensing probe [18-21]. Furthermore, MOFs possessed the potential strength to change the light generation, delivery, and utility because of their design flexibility [22]. Consequently, they are announced as the next generation of classical optical fibers (COFs). The multitude of sensing elements has been employed via utilizing different types of techniques, incorporating fluorescence [23, 24], absorption [25], gratings [26], and the whispering gallery modes [27]. Additionally, the different sorts of light trapping procedures $[28,29]$ are presumably furnishing the auxiliary tracks to enhance the sensing performance in contrast to alternative geometries [30-34]. The potential avenue for sensing based on COFs has been furnished only by the evanescent field due to fundamental impediments affined to their geometrical structures [35-38]. Evanescent field-based MOF sensing platform is of particular interest in context to sensing and detection $[33,37]$. Such a platform's consistency depends critically on the considerable field overlap in air-cladding. It is noteworthy that the easiness for an analyte to be transported via air-holes confers the practicability of such sensing layout $[39,40]$. Structural alternations in photonic cross-section of the MOF can yield an extreme ability to enhance the interaction of guided light with the sample through different configurations and operating principles. Moreover, air-holes may 
hold a sample volume of about nanoliters per $\mathrm{cm}$ of the fiber while still possessing higher sensitivity [41].

Notably, there has been little investigation for fluid sensing based on MOFs due to the low spatial overlap of the light-matter interaction. Nevertheless, reasonable opportunities are possible to improve the evanescent field by adapting the microstructures judiciously [40-43]. Various techniques have been reported for intensifying the fractional power coupled with materials; however, they are appended with intricacy restricted to short optical path length and make the structure more brittle. MOFs have intensified the practical utility of fluid sensing on a larger scale; therefore, techniques have to design for nominating the strength of evanescent field-assisted MOF sensing probes. An alternative approach for evanescent wave sensing is that the light is guided through silica segments among air-holes in a hollow-core MOF cladding [42]. In this way, the infused analyte in all air-holes can be predicted, unlike solid-core MOFs that confine the principle core-mode with the modal area, and only the first ring of air-holes can be affected via evanescent field [43]. One of the powerful postprocessing techniques that can productively enhance the evanescent field is supported by fiber tapering [36]. Robust and the low-loss silica photonic nanowires employing tapered MOF also have been realized [3]. To integrate the tapered fiber's sensitivity with the robustness of COFs, filled-MOF has been nominated due to their strength and the feasibility to offer an excellent sensing platform with better handling. A robust design for aqueous sensing applications for single-mode water-core MOF has been annotated in with almost unity overlap (and loss lower than $1 \mathrm{~dB} / \mathrm{m}$ ) over the extended range of wavelength. By injecting the liquid measurand into air-holes, higher-order coupled cladding-mode enhanced index sensitivity via strong evanescent field-liquid measurand interaction has been recently narrated in [44-47]. A possible limitation could be the compliance associated with infiltration; however, few techniques are evolved to conquer such restrictions.

Polymer-based microstructured optical fibers (P-MOFs) recommend strong predominance over traditional POFs and MOFs. A detailed review of their elementary propagation aspects can be discovered in $[3,44]$. It is worthwhile to articulate that P-MOFs enabled experimentally acceptable platforms (for instance, considering the loss and the ease of fabrication) to tailor the structural parameters (i.e., $d$ and $\Lambda$ ) of the preform and length of the device. Despite that, P-MOFs can be fabricated routinely with a wealth of variety of photonic structures [33]. The superiority of P-MOFs regarding sensing applications over traditional POFs and the MOFs are listed as (1) intrinsic attenuation is potentially more negligible as the light propagates effectively via less material in fiber cross-section (2) they 
can support a single-mode over a vast spectrum of wavelength due to variability in their lightguiding attributes, and (3) the property of single-mode guidance can be attained to enhance the coupling proficiency $[11,13]$. A new strategy has been developed by Cordeiro et al. [30] for the evanescent field-based sensing applications supported by P-MOFs. Further, the feasible designs of dual-core P-MOFs made of Polymethylmethacrylate (PMMA) [7] or Perspex [45] to perform as a highly sensitive label-free biosensor have been explored by Markos et al. [46]. One of the critical advantages of inspecting PMMA (which is a low-priced plastic material extensively used for manufacturing communication grade polymer fibers, and it has a negative thermo-optic coefficient of $\Delta n / \Delta T=-1.1 \times 10^{-4} /{ }^{\circ} \mathrm{C}$ at $\left.0.633 \mu \mathrm{m}[2,47]\right)$ based MOF is provided by their flexibility and inherent strength to directly bind (or to capture selectively) the biomolecules to the surface of holes. Thus, further functionalization can be avoided in contrast to silica fibers [48-50]. A pH sensor comprised of a PMMA fiber coated with a sol-gel to detect phenol red absorbance under various $\mathrm{pH}$ solutions has been documented by Rovati et al. [51]. Later on, a linearly chirped fiber Bragg grating inscribed in PMMA assisted MOF [52] was demonstrated by Korganbayev et al. [6] to enhance the detecting sensitivity.

The numerical simulation of evanescent field overlap with air-holes (of the first inner ring preferably) in solid-core MOF has been performed, showing that a significant fraction of the power could be expended air-holes by cautiously specifying the MOF parameters [39-41]. However, for most of the MOFs fabricated to date, a tiny percentage of the guided mode power penetrates the fiber cladding air-holes. Sensing in MOFs is an exciting dimension of interest, and it is not entirely comprehensible, yet all intimations besmeared with each of the possible geometries. Arif et al. [53] introduced an exotic design of nonlinear MOF (with an elliptical-shaped infiltrated hole in the core) based sensing probe to detect the liquid analytes at the wide range of wavelength $(0.6 \mu \mathrm{m}-1.6 \mu \mathrm{m})$ and did the detailed numerical analysis to examine the impact of design parameters.

In this study, we deem to explore the sensing characteristics of one-rod core PMMA based hexagonal MOF over an extended range of wavelengths by enabling an auxiliary optical model [54]. We quantified the results in terms of sensitivity coefficient (or relative sensitivity coefficient), assuming that the circular holes are filled with water and low index materials (e.g., air/gas). Furthermore, we are focused on calculation and tailoring the parameters of relevant visual traits (such as overlapping factor (OF), quantifying the fraction of optical power exposed to sample) of one-rod core (i.e, one missing air-hole [55]) P-MOFs. 
We introduced an alternative approach to evaluate the confinement loss (CL) of the targeted configurations, possessing a hexagonal assortment of circular air-holes. Furthermore, we dictate the proposing strategies to acquire the modest improvements in the optical performance of evanescent-field-based sensing applications.

We have organized the paper as follows: Section 2 is narrating the theoretical qualities. A strategy for the manifestation of evanescent wave sensing assisted by different configuration of P-MOFs and the simulated results are explored in section 3. Finally, the concluding remarks are briefed in section 4 .

\section{Synopsis of theoretical modeling}

We endorsed an alternative model [54] for an index-guiding one-rod core hexagonal MOF (as illustrated diagrammatically in Fig. 1(a)) and employed it for investigating the elementary light-guiding features of the fiber (as displayed pictorially in Fig. 1(b)). It can be perceived that the solid-core (which is an omitted air-hole) is enclosed by rounded rings of holes (with the inter-hole spacing of $\Lambda$ and diameter $d$ ). The region on the other side of the $5^{\text {th }}$ ring is regarded as uniform. Its effective index is referred as $n_{F S M}$, which is equivalent to the effective mode-index (EMI) of the fundamental space-filling mode [56, 57]. It can be noticed that the model promotes the light guidance via total internal reflection through porous cladding (which assembles round holes arranged in a hexagonal configuration allowing the close confinement of the light in the core) as the average cladding index is sufficiently below than the surrounding. We employed the effective index formulation based on scalar approximation and attained the following characteristics equation [14], described in terms of

$u^{2}=\left(n_{\text {host }}^{2} k_{0}^{2}-\beta_{c l}^{2}\right)$ and $w^{2}=\left(\beta_{c l}^{2}-n_{\text {air }}^{2} k_{0}^{2}\right) \quad\left(\right.$ where $\beta_{c l}=k_{0} n_{F S M} ; k_{0}(=2 \pi / \lambda)$ designates the wave vector in the free-space) on dignifying the boundary conditions and the symmetry distinction (as delineated schematically in Fig. 1(c)).

$$
\begin{aligned}
& \left(Y_{1}(u 0.5 d) \frac{J_{1}(u 0.5 \Lambda)}{Y_{1}(u 0.5 \Lambda)}-J_{1}(u 0.5 d)\right)= \\
& -\left(\frac{w I_{1}(w 0.5 d)}{u I_{0}(w 0.5 d)}\right)\left(Y_{0}(u 0.5 d) \frac{J_{1}(u 0.5 \Lambda)}{Y_{1}(u 0.5 \Lambda)}-J_{0}(u 0.5 d)\right)
\end{aligned}
$$


It is noticeable that one-rod core MOFs possessed the non-Gaussian type of modeprofile; therefore, we have contemplated the following trial field associated with $A, \rho, \rho_{1}$, and $\eta$ as the variables [54];

$$
\Psi_{t}(r, \varphi)=e^{-\rho r^{2}}-A e^{-\rho_{1}\left(r-\eta_{1}\right)^{2}}(1+\cos 6 \varphi)
$$

where $r_{1}=\Lambda$ (see Fig. 1(b)). The mode calculation furnishes the power allocation in the fiber cross-section and the propagation constant (i.e., $\beta_{c o}=k_{0} n_{\text {eff }}$ ) that accumulates as the light propagates. Accordingly, to obtain the enormous value of $\beta_{c o}$ for the dominant core-mode (DCM) of the fiber, we take advantage of MATLAB ${ }^{\circledR}$ Optimization Toolbox.

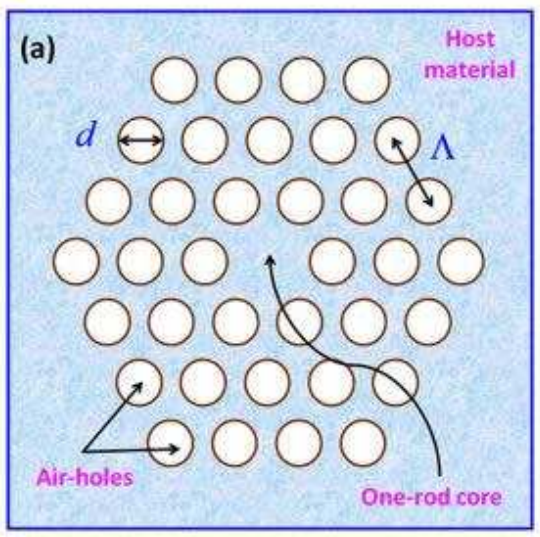

(b)
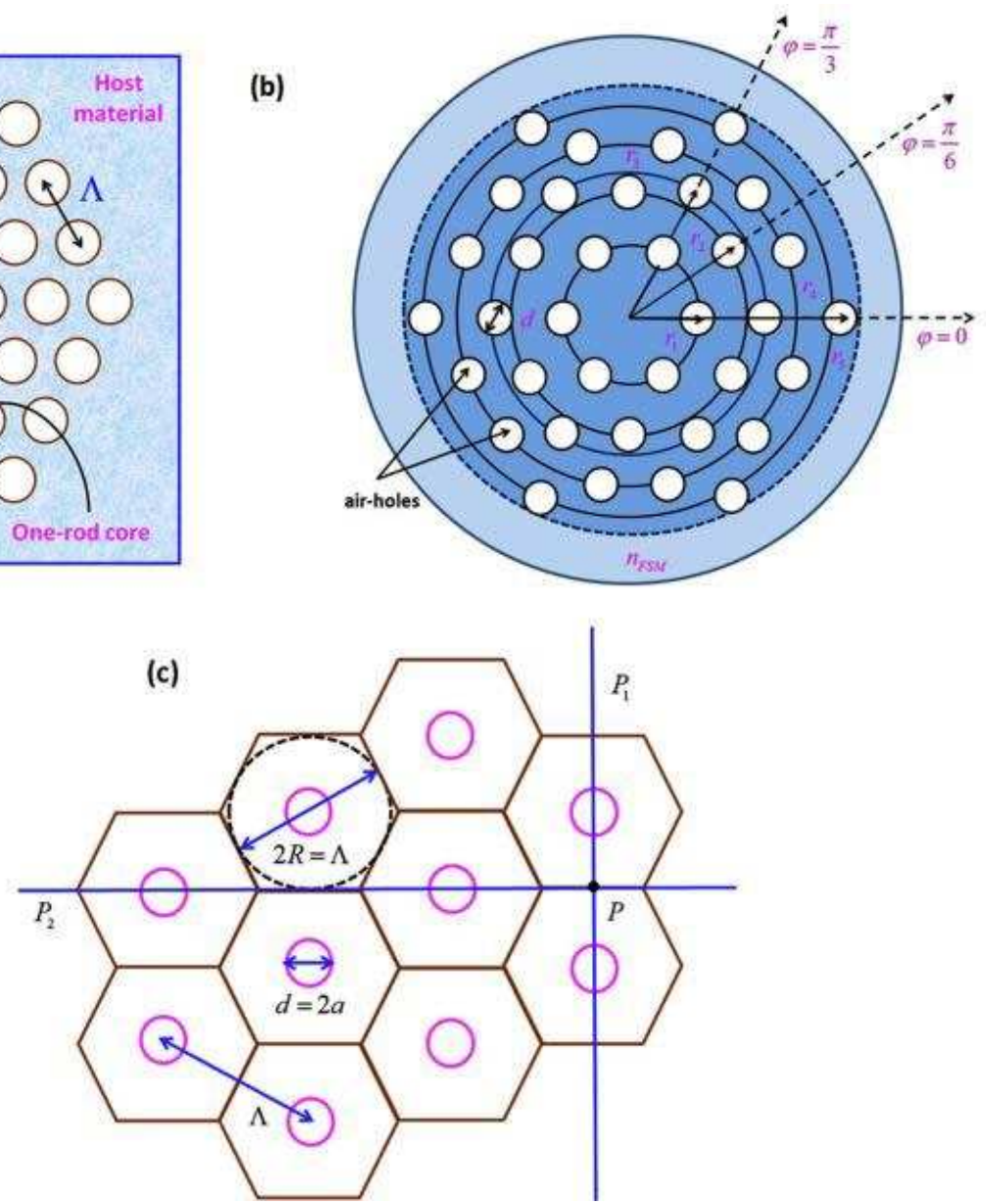

Fig. 1. (a) Schematic representation of the one-rod core MOF (b) simplified sketch of the proposed model, and (c) schematic diagram for cladding structure with hexagonal and commensurate circular unit cell. 
To assess the propagation constant for waveguiding structure, specified by the refractive index (RI) profile $n^{2}(r, \varphi)$, we commenced with the scalar Helmholtz wave equation [58]: $\left(\frac{\partial^{2}}{\partial r^{2}}+\frac{1}{r} \frac{\partial}{\partial r}+\frac{1}{r^{2}} \frac{\partial^{2}}{\partial \varphi^{2}}\right) \Psi_{t}(r, \varphi)+\left(k_{0}^{2} n^{2}(r, \varphi)-\beta_{c o}^{2}\right) \Psi_{t}(r, \varphi)=0$. We object to acquire the immense value for propagation constant by using the following expression [5961]: $\quad \beta_{c o}^{2}=\left(k_{0}^{2} \int_{0}^{2 \pi} \int_{0}^{\infty} n^{2}(r, \varphi)\left|\Psi_{t}(r, \varphi)\right|^{2}-\left(\left|\partial \Psi_{t} / \partial r\right|^{2}+r^{-2}\left|\partial \Psi_{t} / \partial \varphi\right|^{2}\right)\right) d s\left(\int_{0}^{2 \pi} \int_{0}^{\infty}\left|\Psi_{t}(r, \varphi)\right|^{2} d s\right)^{-1} ;$ where $d s(=r d r d \varphi)$ is the cross-sectional area.

It is worth noting that the trial field selection is the cardinal step for fastening impressionability and our methodology's congruence. Additionally, utilizing the introduced model's symmetry attributes, just a sector spanning from $\varphi=0$ to $\varphi=\pi / 6$ is sufficient for the analysis, facilitating to avoid the computation time and cut down the demand of large memory allocations. It is admissible to announce that the vital aspect of auxiliary technique based on the scalar variational principle [58-61] is to furnish a swift and proportionately simple route to enumerate the EMI in contrast to other time- consuming numerical methods.

\section{Simulated results and discourse}

In our simulation, we contemplated PMMA (refractive index $=1.4897$ [52]) based P-MOFs with hexagonal regularity of periodic air-holes in the structure, termed as P-MOF1 $(\Lambda=1.0$ $\mu \mathrm{m}$ and $d / \Lambda=0.90)$ and P-MOF2 $(\Lambda=6.0 \mu \mathrm{m}$ and $d / \Lambda=0.90)$. Our outcomes are expected to provide an extensive literature review to describe the proposed configuration's optical predominance regarding the evanescent sensing.

In MOFs, losses occur due to intrinsic material absorption, structural imperfection, Rayleigh scattering, and micro bending. Despite them, a small fraction of the leakage of light may occur due to the finite number of air-holes in the cladding, which can be quantified in terms of confinement loss [62-67]. Alternatively, it can be defined as the attenuation caused by the geometry of the waveguide. Confinement loss (CL) and sensitivity are two critical guiding competencies of MOF-assisted sensors. Several articles have been published to gain sensitivity at a maximum and CL at a minimum satisfactory sensing application level. In the paper [63], a relative sensitivity coefficient of $\sim 13.23 \%$ has been attained by increasing the inner ring's air-hole diameter and the reduced CL of about $10^{-6} \mathrm{~dB} / \mathrm{m}$ operating at the wavelength of $1.33 \mu \mathrm{m}$. An index-guiding nanostructure MOF has been reported by Olyaee et 
al. [65] and described lower CL, dispersion, and nonlinear effect simultaneously. An improved sensing capacity by developing a new concept for evanescent sensing application in which both core and cladding are porous has been narrated in [30]. The article [66] demonstrated that octagonal MOF possessed a minor loss and higher relative sensitivity coefficient than the hexagonal MOF configuration (with porous core and cladding) for three different analytes.

(a)

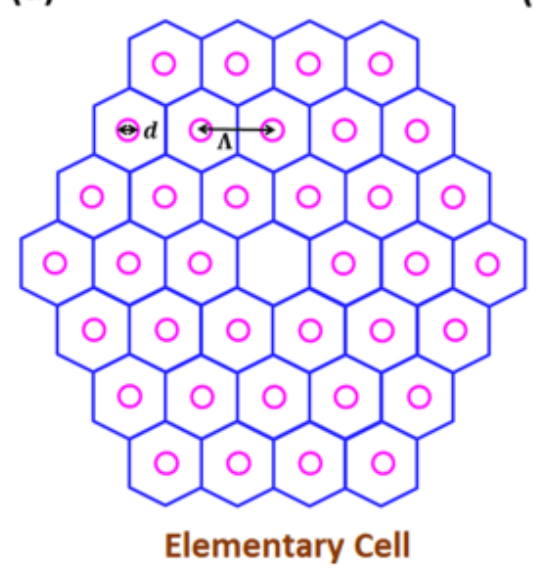

(b)

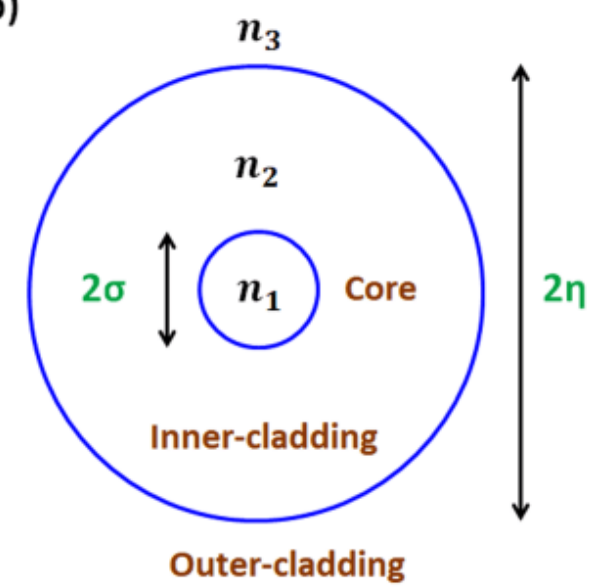

Fig. 2. (a) Schematic sketch for the cross-section of solid-core MOF with finite layers of rounded air-holes (b) corresponding double-clad fiber (DCF) with a depressed index inner cladding.

\subsection{Confinement loss}

Confinement loss (CL) is an additional form of loss that comprehensively occurs in singlematerial fibers induced from the periodic cladding structure. MOFs are usually constituted of pure silica. The guided modes are inherently leaky because the core-index is identical to the outer cladding index without air-holes [39, 41]. Notably, CL associated with a given fiber mode has a direct correlation with its EMI of DCM, which is closely related to the geometrical structure of the MOF. Accordingly, the loss can be optimized by altering the geometry, including air-holes quantity, location, size, and shape.

To introduce the COF theories, MOF with a hexagonal array of circular air-holes of diameter, $d$, and the pitch $\Lambda$ (as illustrated diagrammatically in Fig. 2(a)) can be replaced by the double-clad fiber (DCF) with a depressed index inner cladding (as displayed in Fig. 2(b); where $n_{1}, n_{2}$, and $n_{3}$ are the effective indices of the core, inner cladding, and the outer 
cladding, respectively). Effective core-radius is denoted by $\alpha_{\text {co }}$, and the effective inner cladding radius $\sigma$ is defined so that the area of the inner cladding region $\Omega_{N}$ becomes equal to that of the entire elementary cells (see Fig. 2(a)) as follows: $\Omega_{N}=\pi\left(\eta^{2}-\sigma^{2}\right)=\Omega \sum_{J=1}^{N} 6 J$; where $\Omega=\Lambda^{2}(\sqrt{3} / 2)$ denotes the area of a primitive cell [68], and $N$ is the number of airhole rings. It is noting that the outer cladding index is the same as the core-index, $n_{3}=n_{1}=$ $n_{\text {host }}$ (with $n_{\text {host }}$ being the RI of host material) and assuming that inner cladding index is given by effective index of so-called fundamental space-filling mode (FSM) $[69,70]$ in a hexagonal array of air-holes (i.e., $n_{2}=n_{F S M}$ ).

The power leakage formula is quantified because of the influential $V$-parameter $\left(V=(2 \pi / \lambda) \alpha_{c o} \sqrt{n_{h o s t}^{2}-n_{F S M}^{2}}\right)$ and the practical normalized transverse phase and attenuation constants, i.e., $U\left(=(2 \pi / \lambda) \alpha_{\mathrm{co}} \sqrt{n_{\text {host }}^{2}-n_{\text {eff }}^{2}}\right)$ and $W\left(=(2 \pi / \lambda) \alpha_{\mathrm{co}} \sqrt{n_{e f f}^{2}-n_{F S M}^{2}}\right)$, are defined in the same manner as in the case of COFs and satisfy the subsequent famed relation of $V^{2}=\left(U^{2}+W^{2}\right) \quad[71,72]: \quad 2 \rho_{c}=\left(2 \lambda U^{3} W / \alpha_{c o}^{2} n_{e f f} V^{4} K_{1}^{2}(W)\right) \mathrm{e}^{-2 \eta W / \sigma} ;$ where $\lambda$ is the wavelength, $n_{\text {eff }}$ is the EMI of the principal core-mode propagating in the MOF with rounded air-holes rooted in the cladding region of infinite extent, and $K_{1}$ is the first-order modified Bessel function of the second kind. It is worthwhile to mention that the expression as discussed above can be derived by enabling the perturbation theory (as involved in quantum mechanism [73]), and the details can be found in [72].

Figure 3 describes the variation of confinement loss (in $\mathrm{dB} / \mu \mathrm{m}$ ) with the operating wavelength (in $\mu \mathrm{m}$ ) of the light, for the AF-and-WF configuratons. It demonstrates that the loss increases (due to the spreading of the propagating light) with wavelength growth, for both type of structures. We observed that loss is negligible for AF-and-WF configuration of the P-MOF2. 

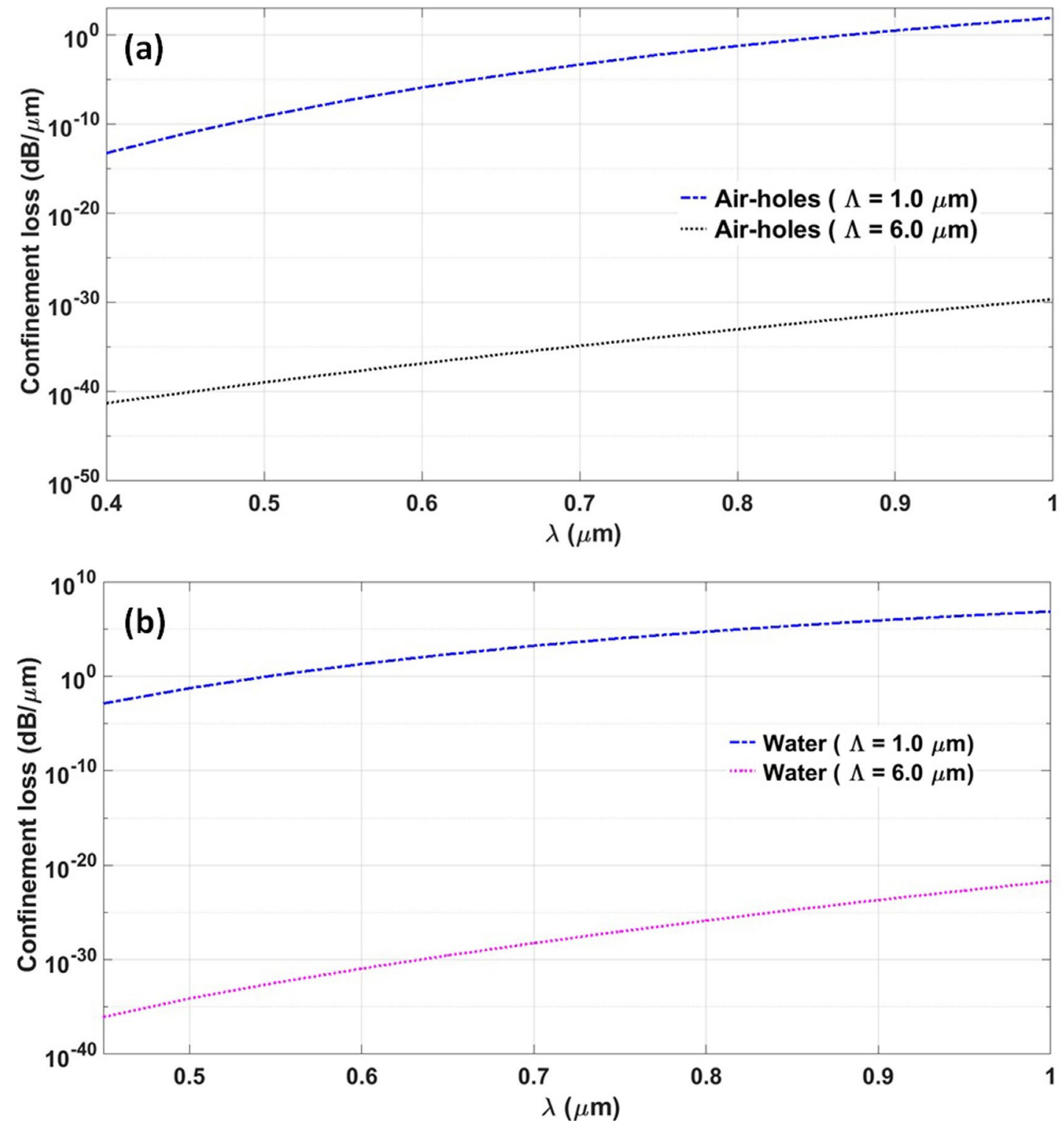

Fig. 3. Spectral dependence of confinement loss for (a) Air-filled and (b) Water-filled configuration of the fibers.

\subsection{Relative sensitivity coefficient}

Sensing different analytes such as protein, alcohol, biochemical, and other higher RI liquids are definitive in science and engineering applications, food processing, industrial applications, and daily life. RI is a constitutive parameter for the recognition of an analyte. MOF discloses an extended range of optical applications (as their optical traits can be enhanced simply by altering the geometrical parameters without variation of materials) due to its unprecedented holey nature, and one of them is sensing [74-76]. Infiltration of the analyte, totally (or selectively) into the holes of MOF, opens a new way of more significant 
interaction between the propagating light and suspected analyte; moreover, it enhances the sensitivity of MOF-based sensing probe multiple times in contrast to $\mathrm{COF}[74,77]$. Measurement of the sensitivity coefficient offers a clear picture of the light-analyte interaction expertise for the MOF.

According to Beer-Lambert law, light is attenuated by the evanescent wave absorption intensity [78, 79]: $I(\lambda)=I_{0}(\lambda) e^{-s_{r} c_{0} l \alpha_{m}}$. The absorption of the targeted sample can be defined by the succeeding relation [40]: $A_{r}=\log \left(I / I_{0}\right)=-s_{r} c_{0} l \alpha_{m}$; where $I$ and $I_{0}$ are the input and output intensities accordingly. The symbol $l$ depicts the length of the fiber and $c_{0}$ is the concentration of the sample. $\alpha_{m}$ is used to designate the absorption coefficient. The sensitivity coefficient is symbolized by $s_{r}$, which is a crucial design parameter employed for quantifying the coupling proficiency of the fiber as a sensing probe, described as $[30,41]$;

$$
s_{r}=\left(n_{r} / n_{\text {eff }}\right) f_{a}
$$

where $n_{r}$ denotes the medium's refractive index to be apprehended and $f_{a}$ represents the percentage of energy coupled to the holes [80].

In the subsequent section, we considered two different configurations of P-MOFs as sensing probes designated as $\mathrm{P}-\mathrm{MOF} 1$ and $\mathrm{P}-\mathrm{MOF} 2$, where holes are assumed to be infiltrated with targeted material, e.g., $\operatorname{air}\left(n_{\text {air }}=1.0\right)$ and water $\left(n_{\text {water }}=1.33\right)$, respectively.

\subsubsection{Air-filled configuration}

Initially, the operating wavelength's impact on the EMI of the principal core-mode is shown in Fig. 4. It is observed that the EMI is monotonically decreasing with increasing wavelength for the analyte to be detected. We reported the typical values of EMI to be 1.449493 (for PMOF1) and 1.488289 (for P-MOF2) at $0.633 \mu \mathrm{m}$ for the air-filled (AF) configuration. The inset depicts the core-mode's near-field intensity pattern (on a logarithm scale) for the fiber's above structure. Furthermore, we have comprised the results based on the finite-element method (FEM) for the introduced model. The values are found in the sequential match to our results, suggesting our designed methodology's robustness.

The influential core size can be articulated in terms of effective mode-area (EMA), which is familiar to play the cardinal role to achieve the efficient modal overlap among the input source and the core-mode of the fiber. Larger core dimensions are capable of supplying 
the high magnitude of overlapping factor (OF) efficiently. The EMA is affined to the productive area of the fiber's core which is computed using the transverse electric field profile of the entire cross-sectional area of the fiber. Nonlinearity is considerately connected to EMA, and it is predominantly inspected as the light carrying region of the fiber, quantified by the following equation [17]: $A_{\text {eff }}=\left(\int_{0}^{2 \pi} \int_{0}^{\infty}\left|\Psi_{2}(r, \varphi)\right|^{2} d s\right)^{2} /\left(\int_{0}^{2 \pi} \int_{0}^{\infty}\left|\Psi_{2}(r, \varphi)\right|^{4} d s\right)$.

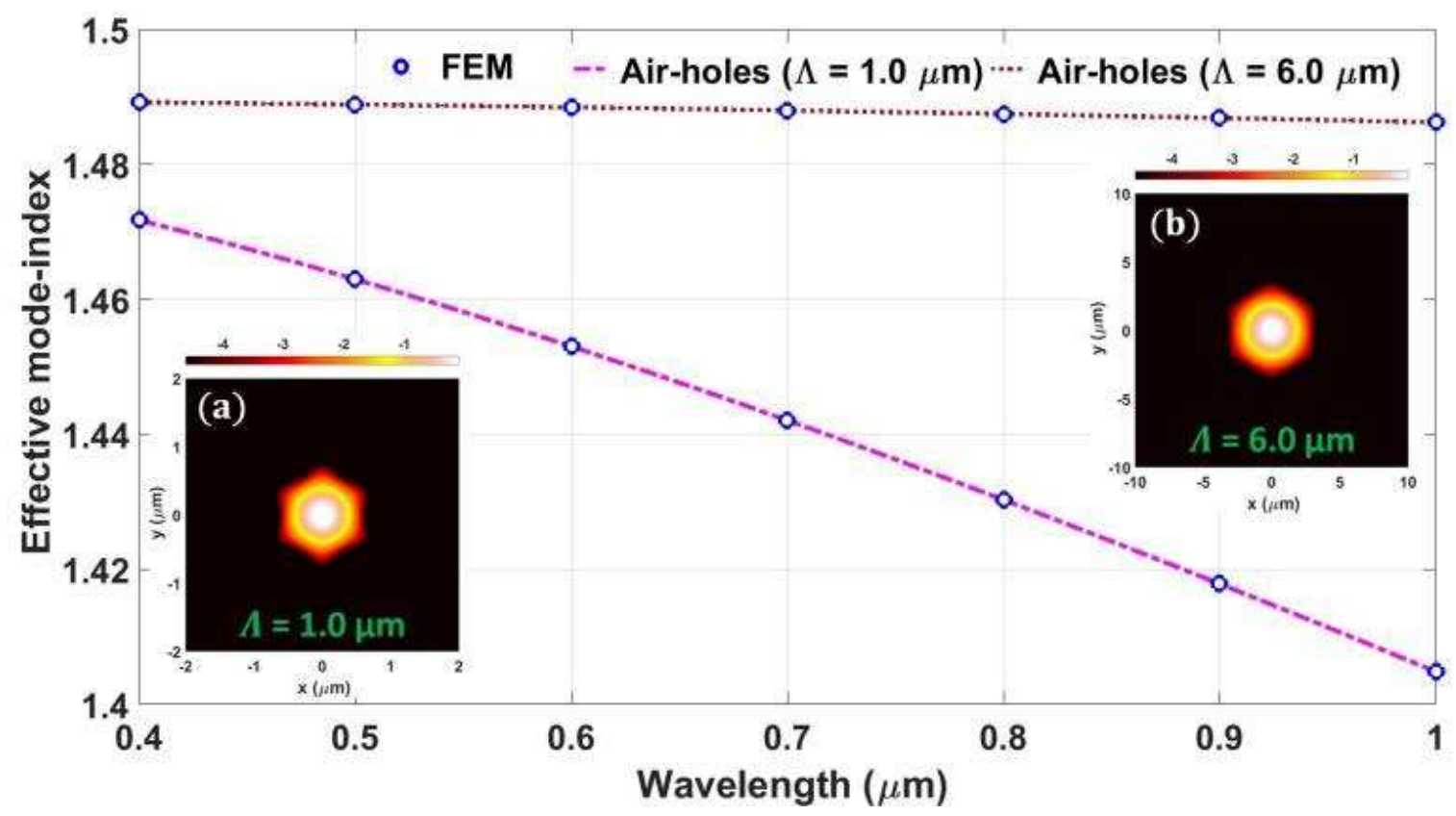

Fig. 4. Spectral dependence of the effective mode-index against wavelength for the AFconfiguration. The inset demonstrates their near-field intensity pattern on the logarithm scale.

The graphical representation of EMA for the DCM of the P-MOFs (in case of AFconfiguration) is shown in Fig. 5 for the wavelength range of $0.4-1.0 \mu \mathrm{m}$. It is observable that EMA for P-MOF2 is designed in such a way to promote its sensing attributes. Inset delineates the $2 \mathrm{D}$ surface plot for the modal field of the proposed geometry. We reported the EMA of $0.71 \mu \mathrm{m}^{2}$ and $16.39 \mu \mathrm{m}^{2}$ for P-MOF1 and P-MOF2, respectively, at the wavelength of interest $0.633 \mu \mathrm{m}$.

The spectral dependence of $s_{r}$ over the PMMA [81] transparency domain is employed for simulation and evaluated for the geometrical configuration, e.g., when all holes are supposed to be infiltrated with air. It is evident from Fig. 6 that $s_{r}$ is steadily increasing with the increase in wavelength (from $0.4 \mu \mathrm{m}$ to $1.0 \mu \mathrm{m}$ ). Inset in Fig. 5(a)-(b) illustrates the 
simulated near-field pattern of DCM with a colour bar at $0.633 \mu \mathrm{m}$ for the AF-configuration of P-MOF1 and P-MOF2, respectively. It can be inferred that the fiber geometry is deployed on the hexagonal pattern. In contrast, the core delineates the circular-like shape (which is analogous to linearly polarized $L P_{01}$ principal core-mode of the COF).

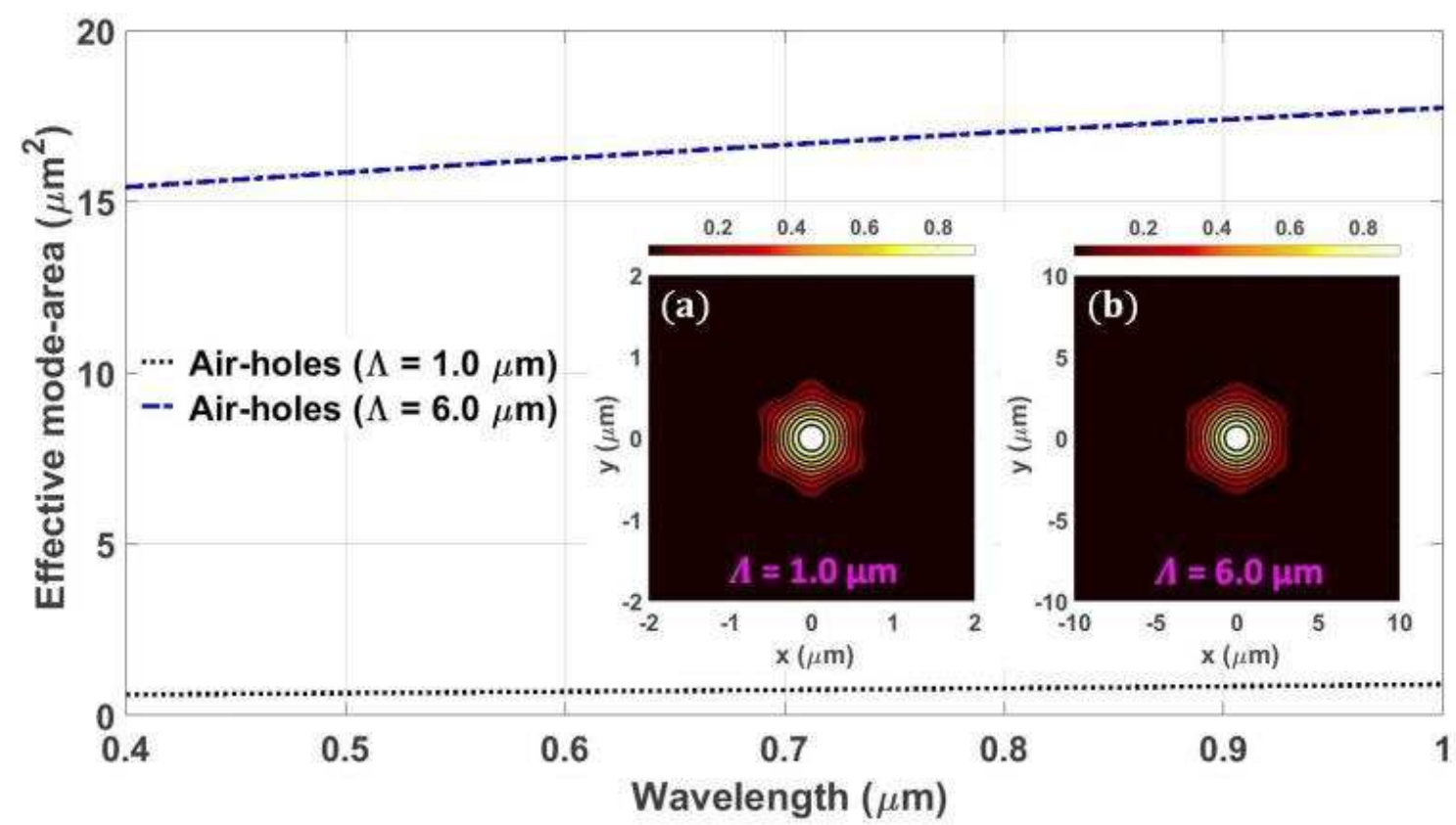

Fig. 5. Effective mode-area against wavelength for AF-configuration of the introduced PMOFs and the inset depicts their 2D surface plot for intensity distribution.

If holes are infiltrated with air, then $s_{r}$ assume the values of $0.9 \%$ (for P-MOF2) and $5.7 \%$ (for P-MOF1) at $0.633 \mu \mathrm{m}$. Higher sensitivity results in tight confinement of the light in the core region (resulting in smaller EMA, see Fig. 5) and the higher electromagnetic power interaction among light and the measurand. From the results, it can also be visualized that increment of sensitivity is governed by confinement loss. This phenomenon highlights the significant interaction between the analyte and the light at a higher wavelength realm.

It is noteworthy that such configurations can provide sufficiently higher sensitivity while using larger air-holes, permitting quicker filling and amalgamation of the particles [82]. Furthermore, it is argued that P-MOFs can facilitate $s_{r}$ as accomplished for microstructuredcore MOF $[30,77]$; however, $s_{r}$ is restricted by small core dimension (which is quantified in terms of EMA) of the traditional MOF, demanding the correctness during the process of alignment. Besides, it reduces the modal overlap between MOF and COF. At this end, it will be favourable to narrate that a bunch of tiny holes in the core may increase the sensitivity 
more, but it will affix fabrication complexity. Considering the reduction of fabrication complexity and maintaining the impassable trade-off among sensitivity and the confinement loss, we have to tolerate extra afford to optimize the core alignment. Therefore, we are concerned to examine the input beam's coupling proficiency [30] to the MOF's core-mode by evaluating the overlapping factor [83] in the next section.

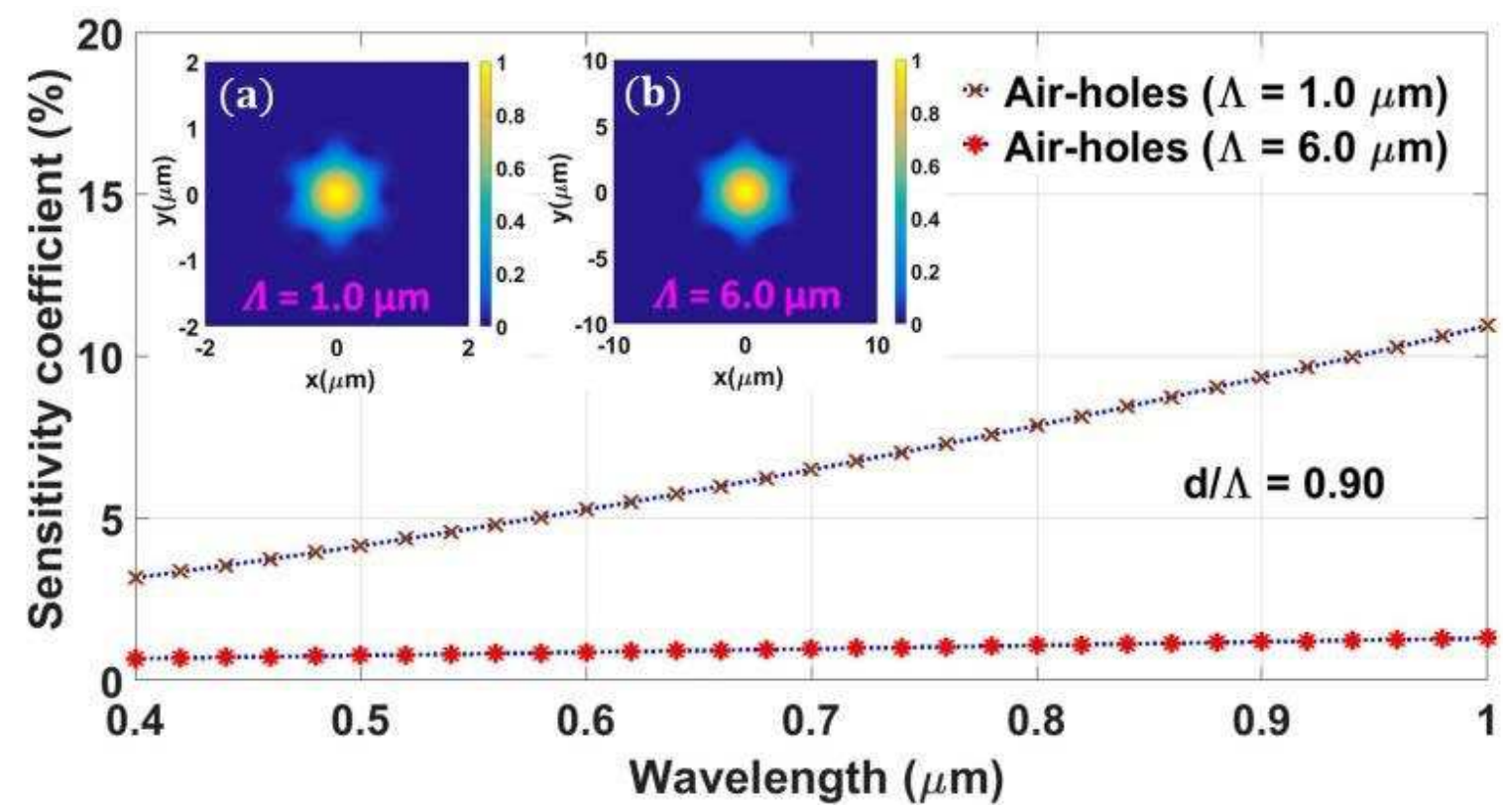

Fig. 6. Sensitivity coefficient against wavelength for two different cladding configurations of P-MOFs. Inset depicts near-field of cardinal core-mode at $\Lambda=1.0 \mu \mathrm{m}$ and $6.0 \mu \mathrm{m}$, when holes are assumed to be infiltrated with air.

The overlapping factor (OL) is evaluated by employing the under-mentioned overlap integral [84-86];

$$
\eta_{o}=\left(\left|\int_{0}^{2 \pi} \int_{0}^{\infty} \Psi_{1}(r, z) \Psi_{2}(r, \varphi) d s\right|^{2}\right)\left(\left(\int_{0}^{2 \pi} \int_{0}^{\infty}\left|\Psi_{1}(r, z)\right|^{2} d s\right)\left(\int_{0}^{2 \pi} \int_{0}^{\infty}\left|\Psi_{2}(r, \varphi)\right|^{2} d s\right)\right)^{-1}
$$

where $\Psi_{1}(r, z)$ denotes the field-profile of a Gaussian beam and $\Psi_{2}(r, \varphi)$ represents the mode-profile for the lowest-order core-mode of the P-MOF. The field-profile of the input beam can be manifested in beam radius $w(z)=w_{0} \sqrt{1+\left(2 z / k_{0} w_{0}^{2}\right)^{2}}$, the radius of curvature 
$R(z)=z\left(1+\left(k_{0} w_{0}^{2} / 2 z\right)^{2}\right)$, and the phase $\varphi_{0}(z)=\tan ^{-1}\left(\lambda z / \pi w_{0}^{2}\right)$, after propagating over a distance, $z$ as [87-89]: $\Psi_{1}(r, z)=(\sqrt{2 / \pi} / w(z)) \exp \left(-\left(\frac{r^{2}}{w^{2}(z)}\right)-\left(\frac{i \pi r^{2}}{\lambda R(z)}\right)+i \varphi_{0}(z)-i k_{0} z\right)$.

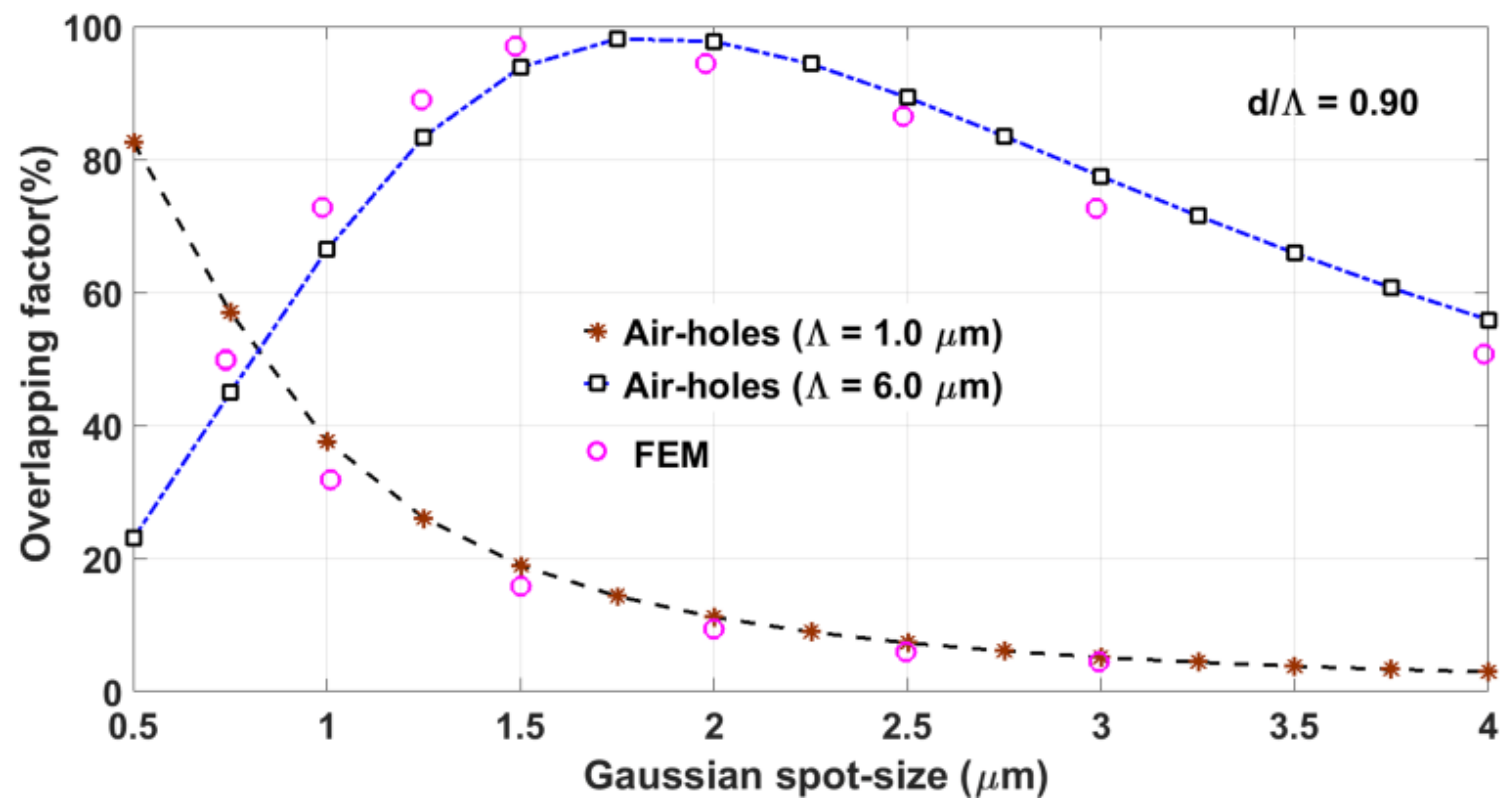

Fig. 7. Overlapping factor against Gaussian spot-size when it is assumed that all P-MOF holes are filled with air.

The simulated results are demonstrated in Fig. 7 at $0.633 \mu \mathrm{m}$ depicting the variation of OF against the Gaussian spot-size (GSS) for AF-configuration. We reported the OF $\sim 19 \%$ (for P-MOF1) and $\sim 94 \%$ (for P-MOF2) for the input beam of GSS (= $1.5 \mu \mathrm{m}$ ). It can be inferred that a higher value of OF for P-MOF2 is being furnished by its DCM (which looks like a Gaussian mode and has large EMA (see Fig. 5)), leading to maximum overlapping with the input field-profile. Notably, the modal OF falls to less than $20 \%$ in the case of P-MOF1. Consequently, we would like to communicate that such configuration demands an auxiliary route to achieve the more substantial light-fluid overlap.

For scrutinizing the theoretically simulated result's genuineness, we comprised the numerical results based on finite-element method (FEM) as quoted from [30]. The sequential matching among the results is noticed. Thus, it is argued that adequate correctness can be availed by employing our approximate method, which further alleviates the challenge for high memory allotment and computation time. 
Table 1. Relative errors in overlapping factor for AF-configuration of P-MOFs

\begin{tabular}{|c|c|c|c|}
\hline $\begin{array}{c}\text { Gaussian spot-size }(\mu \mathrm{m}) \\
\text { P-MOF1 }\end{array}$ & $\begin{array}{c}\eta_{01}(\text { Optical } \\
\text { Model })\end{array}$ & $\begin{array}{c}\eta_{02}(\text { Quoted } \\
\text { Data) [30] }\end{array}$ & $\Delta \eta_{0}(\%)=\left|\frac{\left(\eta_{01}-\eta_{02}\right)}{\eta_{02}}\right| \times 100$ \\
\hline 1.0 & 37.665 & 32.887 & 14.53 \\
\hline 2.0 & 11.157 & 10.349 & 7.81 \\
\hline 3.0 & 5.127 & 4.981 & 2.93 \\
\hline $\begin{array}{c}\text { Gaussian spot-size }(\mu \mathrm{m}) \\
\text { P-MOF2 }\end{array}$ & $\eta_{01}($ Optical & $\eta_{02}($ Quoted \\
Model $)$ & Data) $[30]$ & $\Delta \eta_{0}(\%)=\left|\frac{\left(\eta_{01}-\eta_{02}\right)}{\eta_{02}}\right| \times 100$ \\
\hline 1.0 & 66.456 & 71.788 & 7.43 \\
\hline 2.0 & 97.768 & 95.491 & 2.38 \\
\hline 3.0 & 77.456 & 74.613 & 3.81 \\
\hline
\end{tabular}

After initiating the proposed model's performance graphs, it is required to probe the design's closeness. Therefore, to express our theoretical result's reliability, we calculated the relative error: $\Delta \eta_{0}(\%)=\left|\left(\eta_{01}-\eta_{02}\right) / \eta_{02}\right| \times 100$ for the GSS of $1.0 \mu \mathrm{m}, 2.0 \mu \mathrm{m}$, and $3.0 \mu \mathrm{m}$ at $\lambda=0.633 \mu \mathrm{m}$ (see Table 1 ), where $\eta_{01}$ is obtained from the anticipated model and $\eta_{02}$ is quoted from [30].

\subsubsection{Water-filled configuration}

Recently, the speculation of infiltrating MOF holes with different materials (e.g., gasses and liquids) has provided a revolutionary route for chemical and biological sensing [77]. Monro et al. [90] have introduced an MOF structure for the evanescent field-assisted sensing applications whereby both core and cladding are microstructured (facilitates the enhancement of electromagnetic power interaction with the sample to be sensed). Using such MOF designs, higher $s_{r}$ (with low CL) for the targeted analytes has been documented [91].

In this consecutive section, we object to study the sensing peculiarities in terms of water as targeted material. Notably, the underlying problem is analogous and similar designs can be implemented as gas sensors. It is possible to achieve robust index-guidance by surrounding a water-core with an air/silica microstructured cladding, well confined to the core and sizeable modal OF. Additionally, such structures can be designed to be multi- 
moded, single-moded (or few-moded), as required by the measurement system. To attain robust confinement in a water core, the average index of the air-silica cladding must be below the water index. Intuitively, this average index will be lower enough when the air-filling fraction is high (proportional to $\left.(d / \Lambda)^{2}\right)$ and for a sufficiently longer normalized wavelength $(\lambda / \Lambda)$. The underlying operating principle is the same, but the details must incorporate water's material characteristics. Since the RI of water is considerably higher than that of the air; therefore, different types of designs are also possible.

In Fig. 8, we have explored the mode-index for DCM of the P-MOF's water-filled (WF) configuration. The inset demonstrates their near-field intensity pattern on a logarithm scale. We documented EMI of 1.459482 and 1.488984, respectively, for WF-configuration at $0.633 \mu \mathrm{m}$. For checking our result's consistency, we have included those based on the FEM (as displayed by solid circles), and the coherent matching between them announces our simplified model's competence.

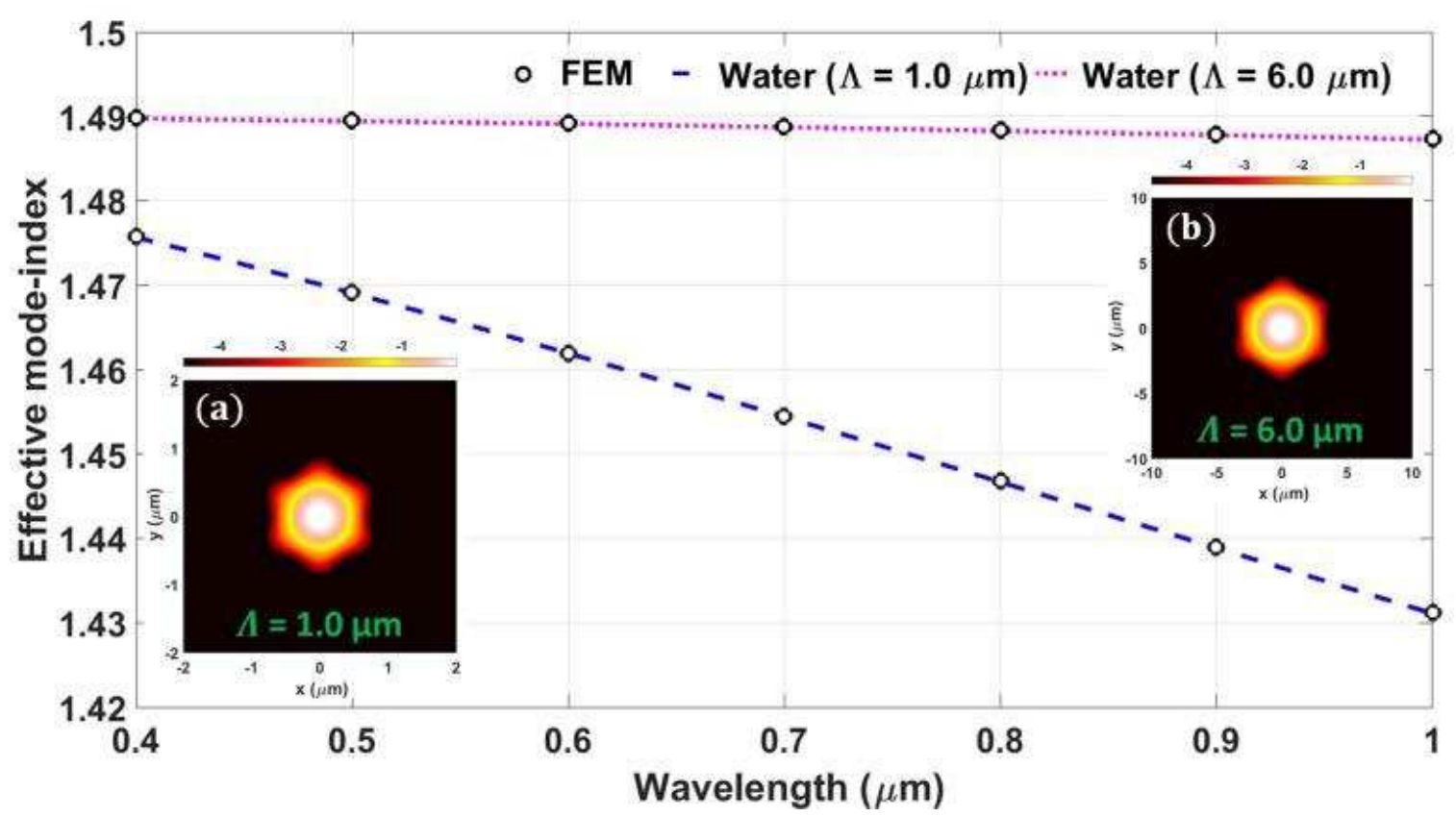

Fig. 8. Effective mode-index against wavelength for WF-configuration of the fibers. Inset illustrates their near-field intensity pattern on a logarithm scale.

The graphical representation of EMA for the DCM of the P-MOFs (in the case of WF-configuration) is shown in Fig. 9. Inset portrait their 2D surface plot for the modal field of the proposed geometry. The EMA of $0.98 \mu \mathrm{m}^{2}$ and $21.62 \mu \mathrm{m}^{2}$ at $0.633 \mu \mathrm{m}$ for the said configurations is also articulated. 


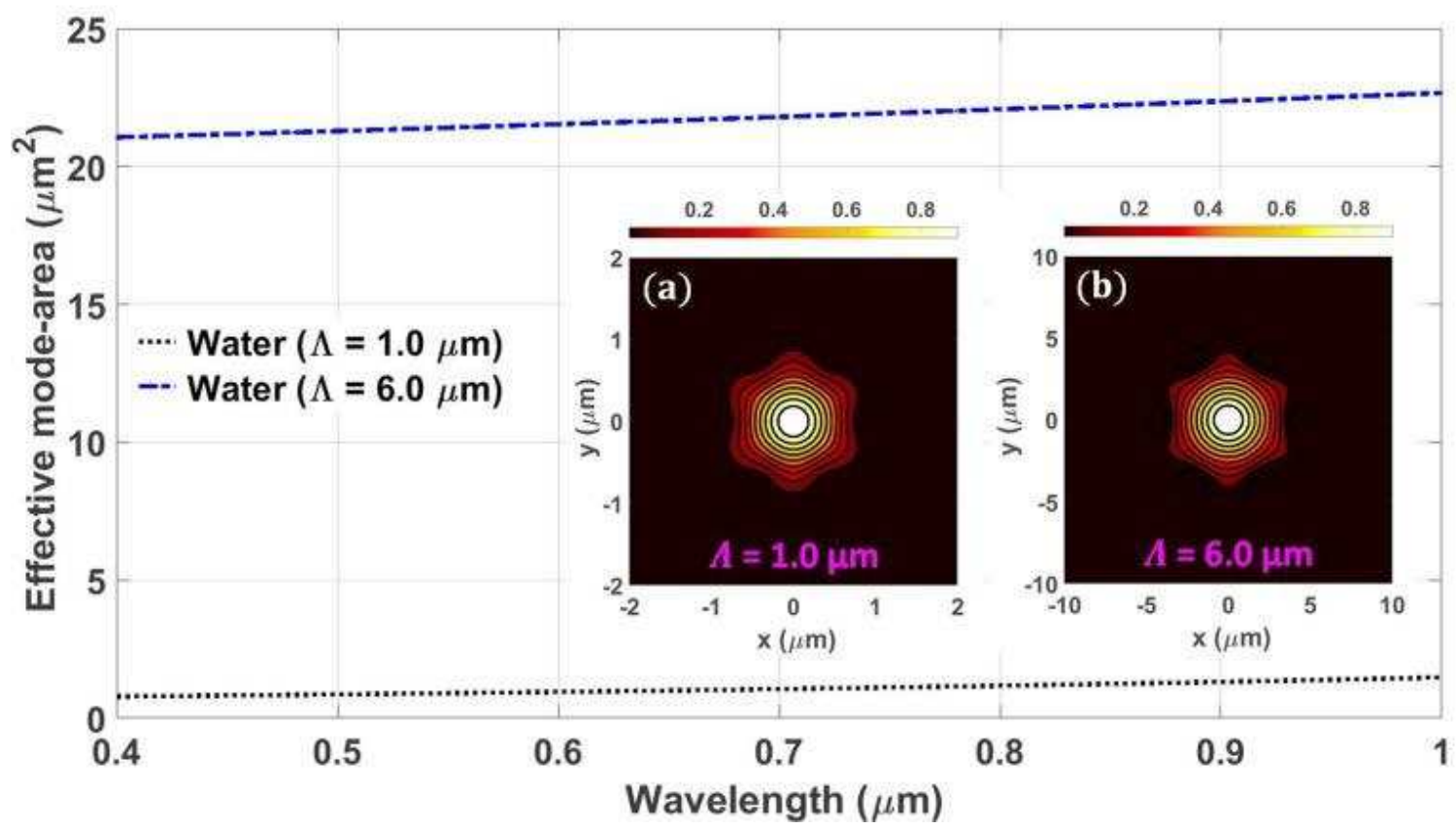

Fig. 9. Effective mode-area as a function of wavelength for water-filled configuration of the introduced P-MOFs.

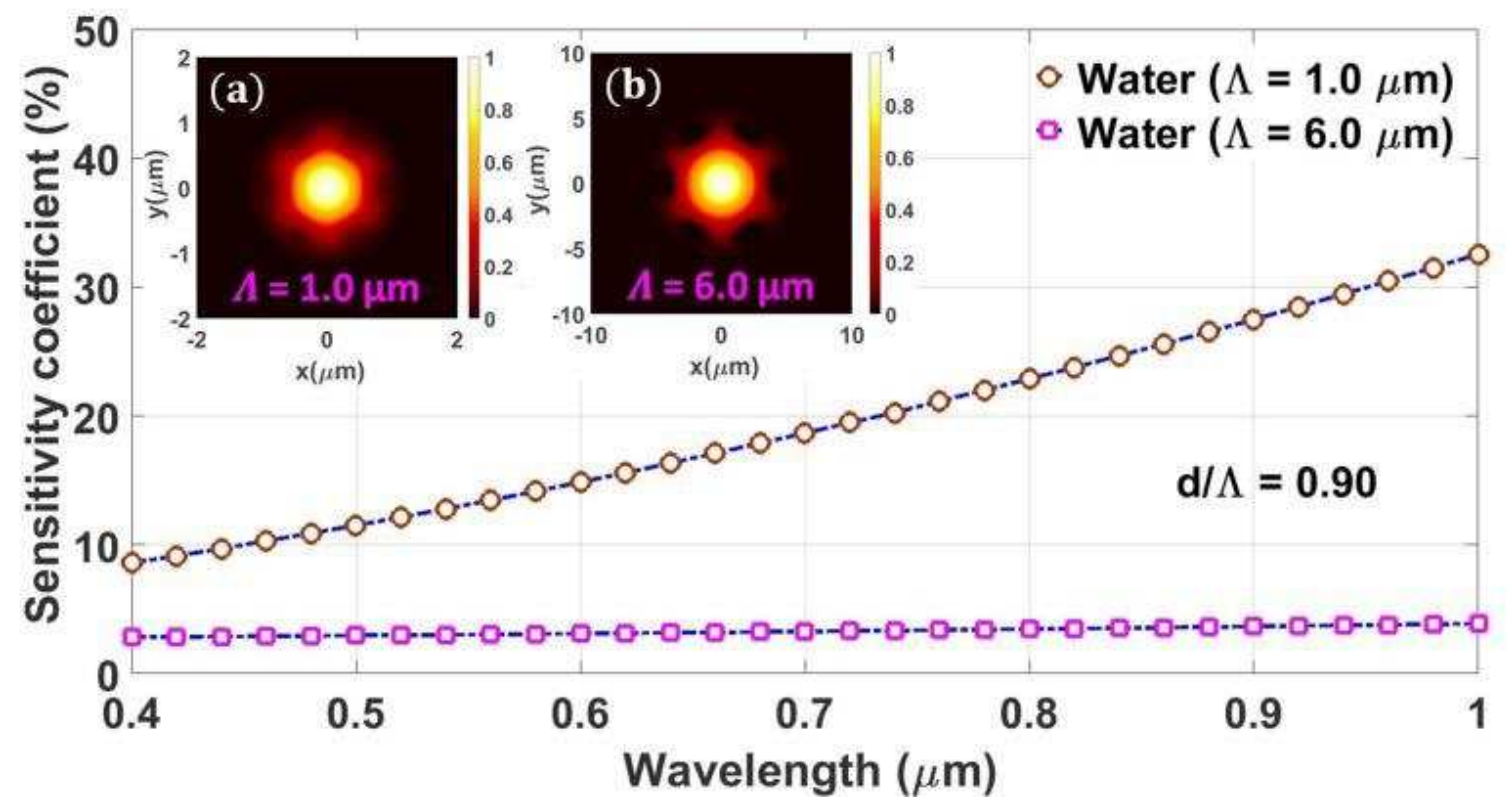

Fig. 10. Sensitivity coefficient against wavelength for WF-layout. Inset depicts their nearfield patterns at $0.633 \mu \mathrm{m}$.

Figure 10 shows the sensitivity coefficient against wavelength $(0.4 \mu \mathrm{m}-1.0 \mu \mathrm{m})$ for the fiber's WF-configuration at $d / \Lambda=0.90$. It is visual that $s_{r}$ yield to $3.2 \%$ (for P-MOF2) and $16.1 \%$ (for P-MOF1) at $\lambda=0.633 \mu \mathrm{m}$. Inset depicts the near-field pattern for DCM of 
the targeted fibers, respectively. It can be seen that the modal field is well-confined in the WF-configuration of the P-MOF2. Furthermore, it is observed that with increasing EMA (see Fig. 9), $s_{r}$ starts growing because of the high degree of modal spreading due to the large light to analyte interaction. Since the light is guided via total internal reflection; therefore, the effect of RI contrast of the core material and the analyte is more pronounced on the light guidance attributes. Besides, this would also affect the $s_{r}$ as it is the analyte's power accumulation capability relative to the total power propagated throughout the cross-section.

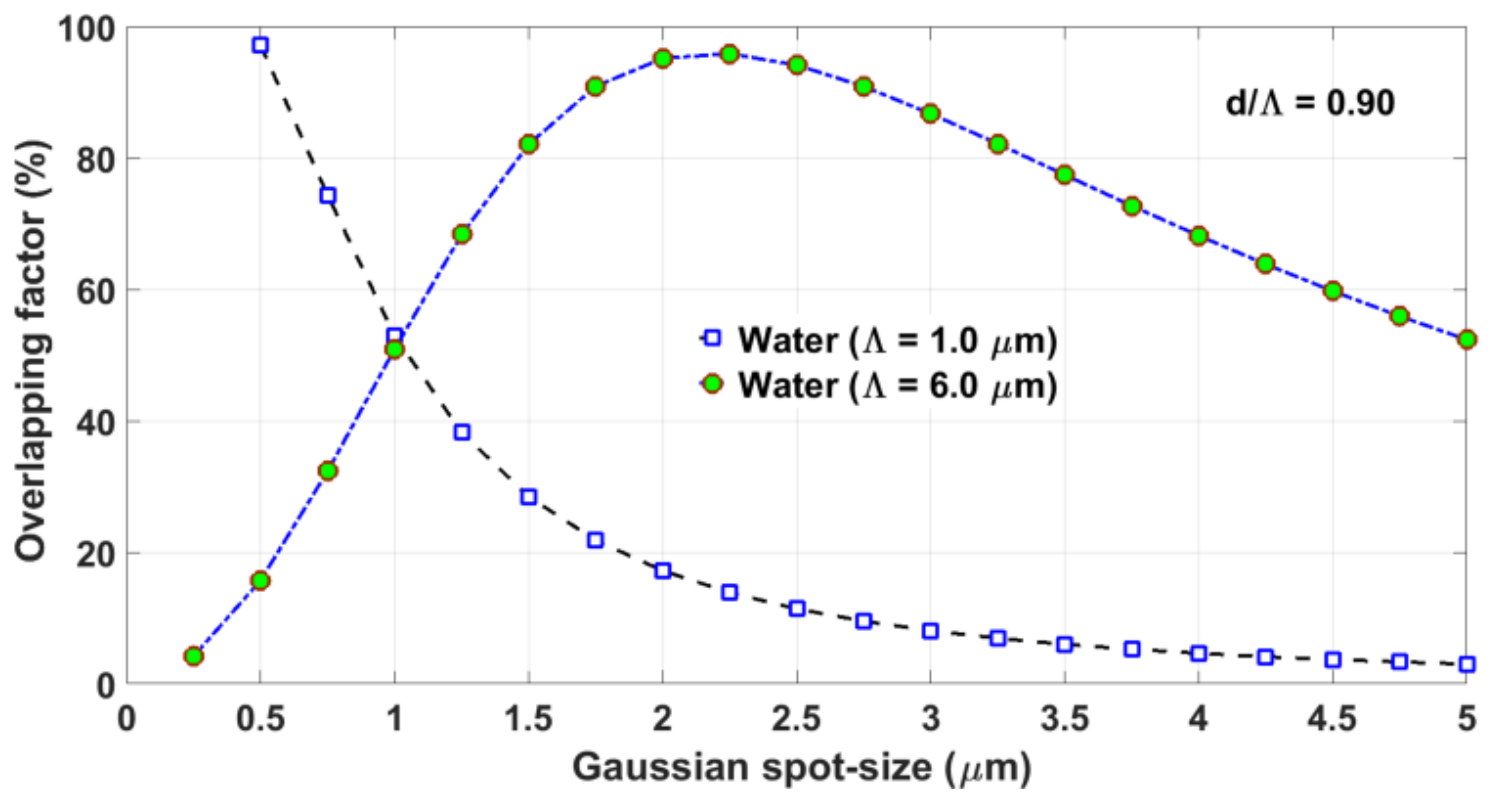

Fig. 11. Coupling efficiency against Gaussian radius between Gaussian optical mode and the core-mode of P-MOF's water-filled design at $0.633 \mu \mathrm{m}$.

When all holes are filled with water, the observed trend of variation as shown in Fig. 11 , which is analogous to as delineated in Fig. 7 , and the modal OF $\sim 29 \%$ and $\sim 82 \%$ is obtained for P-MOF1 and P-MOF2 respectively. On the contrary, water/PMMA configuration of MOF (with $\Lambda=6.0 \mu \mathrm{m}$ and $d / \Lambda=0.90$ ) have OF lower than $94 \%$ for GSS of $1.5 \mu \mathrm{m}$ (in contrast to the previous case). Further, we would like to mention that for PMOF1, the modal OF more than $19 \%$ is achieved (compared to the earlier point) by employing an input beam of GSS, suggesting a prompted route to enhance the substantial light-fluid overlap. We have tabulated the typical values of overlapping factor (\%) for airand-water-filled configurations of hexagonal P-MOF (see Table 2). 
It is worth highlighting that the sample to be studied is explored through the evanescent field, which falls significantly from the fiber surface; therefore, the measurand can be sensed near the interface of holes (or even deployed as a thin layer) [92-94]. Thus, it is anticipated that a well-designed WF-configuration with high OF and low loss can directly improve the sensitivity and the optical power requirement. In a recent study, it is interesting to highlight that higher sensitivity and lower CL of MOF for sensing applications also have been attempted by using an octagonal cladding structure [78]. It is suggested that 5-ring octagonal MOF can have higher sensitivity and lower CL [79]; however, in practical manufacturing, such structure requires extra capillaries than hexagonal structure. Keeping a large number of capillaries will make the high cost to fabricate. In this frame of reference, sensing using the hexagonal channel of air-holes may reduce the structure's complexity.

Table 2. The overlapping factor (\%) for WF-configuration at $0.633 \mu \mathrm{m}$

\begin{tabular}{|c|c|c|c|}
\hline Gaussian spot-size $(\mu \mathrm{m})$ & 0.5 & 2.5 & 4.5 \\
\hline P-MOF1 $(\Lambda=1.0 \mu \mathrm{m} \& d / \Lambda=0.90)$ & 97.14 & 11.39 & 3.67 \\
\hline P-MOF2 $(\Lambda=6.0 \mu \mathrm{m} \& d / \Lambda=0.90)$ & 15.75 & 94.15 & 59.75 \\
\hline
\end{tabular}

To this end, we would like to narrate that a novel strategy for P-MOF assisted sensing design is demonstrated with potential advantages in contrast to previously reported, and the qualitative description for water-core fiber is also documented. By employing the same basic strategy, we are assured that fibers can be readily designed to accommodate the different sample materials, interaction wavelengths, and core sizes. Furthermore, a high-performance temperature sensor can be realized by utilizing the selectively filled and the solid-core MOF with a central air-bore [93]. We affirmed that our approximate method could provide a new dimension for exploring the evanescent sensing based usage in P-MOFs.

\section{Conclusion}

Evanescent sensing-based merits of the one-rod core hexagonal P-MOF are being investigated using a simplified theoretical model. The likelihood to enhance the coupled power is elucidated for different configurations of the fibers. We reported the overlapping factor of $\sim 94 \%$ (sensitivity coefficient of $\sim 0.9 \%$ ) and $\sim 82 \%$ (sensitivity coefficient of $\sim$ 
$3.2 \%$ ) for AF-and-WF configuration of P-MOFs, respectively, at the applied wavelength of $0.633 \mu \mathrm{m}$. We anticipate that WF-configuration can have an immediate impact on optical sensing. For the expected model confinement loss, effective mode-index and mode-area of the fundamental core-mode are also evaluated. Our recommended model provided a higher overlapping factor with tighter confinement of optical energy; therefore, it can successfully overcome the critical trade-off between confinement loss and sensitivity. We made comparisons for inspecting the simulated result's sustainability, and a sequential appendage among the results is outlined.

The proposed structure has better guiding capability, and the manufacturing of this type of geometry is possible with the current state-of-the-art nanofabrication techniques. We are concerned about exploiting the input/output coupling proficiency to such various structures and exploring the feasibility that similar design strategies may further enhance their optical power delivery performance. Also, we are assertive that optimizing such systems by utilizing our analytical approach can enlarge the evanescent field's magnitude, unifying the juncture of employing fibers with the suspended-core [94]. We believe that our introduced model can offer great potentiality for evanescent sensing applications; moreover, it furnishes outstanding futuristic research obligations.

\section{Acknowledgments}

D.K. Sharma is thankful to IIT Kanpur, Kanpur (U.P), India, to furnish the Institute Postdoctoral Fellowship (PDF-102). The author is grateful to Prof. A. Sharma, IIT Delhi, New Delhi, India, for his worthwhile discussion.

\section{References}

[1] M. Pesavento, L. Zeni, L.De Maria, G. Alberti, and N. Cennamo, "SPR-optical fibermolecularly imprinted polymer sensor for the detection of furfural in wine," Biosensors, $11,1-12(2021)$.

[2] G. Emiliyanov, J.B. Jensen, O. Bang, P.E. Hoiby, L.H. Pedersen, E.M. Kjær, and L. Lindvold, "Localized biosensing with Topas microstructured polymer optical fiber," Opt. Lett., 32, 460-462 (2007).

[3] K. Peters, "Polymer optical fiber sensors - a review," Smart Mater. Struct., 20, 013002, (2011).

[4] J. Zubia and J. Arrue, "Plastic optical fibers: an introduction to their technological processes and applications," Opt. Fiber Technol., 7, 101-140 (2001). 
[5] A. Leal-Junior, A. Frizera-Neto, C. Marques, and M.J. Pontes, “A polymer optical fiber temperature sensor based on material features," Sensors, 18, 301 (2018).

[6] S. Korganbayev, R. Min, M. Jelbuldina, X. Hu, C. Caucheteur, O. Bang, B. Ortega, C. Marques, and D. Tosi, "Thermal profile detection through high-sensitivity fiber optic chirped Bragg grating on microstructured PMMA fiber,” J. Light. Technol., 36, 47234729 (2018).

[7] X. Cheng, J. Bonefacino, B.O. Guan, and H.Y. Tam, "All-polymer fiber-optic pH sensor," Opt. Express, 26, 14610-14616 (2018).

[8] R. Min, B. Ortega, and C. Marques, "Fabrication of tunable chirped mPOF Bragg gratings using a uniform phase mask," Opt. Express, 26, 4411-4420 (2018).

[9] D.J. Webb, "Fiber Bragg grating sensors in polymer optical fibers," Meas. Sci. Technol. 26, 092004 (2015).

[10] C.A.F. Marques, D.J. Webb, and P. Andre, "Polymer optical fiber sensors in human life safety," Opt. Fiber Technol., 36, 144-154 (2017).

[11] A. Leal-Junior, A. Frizera, M.J. Pontes, A. Fasano, G. Woyessa, O. Bang, and C.A.F. Marques, "Dynamic mechanical characterization with respect to temperature, humidity, frequency and strain in mPOFs made of different materials," Opt. Mater. Express, 8, 804815 (2018).

[12] A. Leal-Junior, A. Frizera-Neto, C. Marques, and M.J. Pontes, "Measurement of temperature and relative humidity with polymer optical fiber sensors based on the induced stress-optic effect," Sensors, 18, 916 (2018).

[13] A.G. Leal-Junior, A. Frizera, R. Min, M.J. Pontes, A. Fasano, G.T. Woyessa, O. Bang, and C. Marques, "Influence of the cladding structure in PMMA mPOFs mechanical properties for strain sensors applications,” IEEE Sens. J., 18, 5805-5811 (2018).

[14] P.St.J. Russell, “Photonic crystal fibers,” J. Light. Technol., 24, 4729-4749 (2006).

[15] T.A. Birks, J.C. Knight, and P.St.J. Russell, "Endlessly single-mode photonic crystal fiber," Opt. Lett., 22, 961-963 (1997).

[16] H. Ademgil and S. Haxha, "Endlessly single mode photonic crystal fiber with improved effective mode area," Opt. Commun., 285, 1514-1518 (2012).

[17] T. Yang, E. Wang, H. Jiang, Z. Hu, and K. Xie, "High birefringence photonic crystal fiber with high nonlinearity and low confinement loss," Opt. Express, 23, 8329-8337 (2015).

[18] T.M. Monro, D.J. Richardson, and P.J. Bennet, "Developing holey fibers for evanescent field devices,” Electron. Lett., 35, 1188-1189 (1999). 
[19] J.B. Jensen, L.H. Pedersen, P.E. Hoiby, L.B. Nielsen, T.P. Hansen, J.R. Folkenberg, J. Riishede, D. Noordegraaf, K. Nielsen, A. Carlsen, and A. Bjarklev, "Photonic crystal fiber based evanescent-wave sensor for detection of biomolecules in aqueous solutions," Opt. Lett., 29, 1974-1976 (2004).

[20] L. Rindorf, P.E. Hoiby, J.B. Jensen, L.H. Pedersen, O. Bang, and O. Geschke, "Towards biochips using microstructured optical fiber sensors," Anal. Bioanal. Chem., $385,1370-1375$ (2006).

[21] M.E. Bosch, A.J.R. Sanchez, F.S. Rojas, and C.B. Ojeda, "Recent development in optical fiber biosensors," Sensors, 7, 797-859 (2007).

[22] X. Fan, I.M. White, S.I. Shopova, H. Zhu, J.D. Suter, and Y. Sun, "Sensitive optical biosensors for unlabeled targets: A review," Anal. Chim. Acta, 620, 8-26 (2008).

[23] S.C. Warren-Smith, S. Heng, H. Ebendorff-Heidepriem, A.D. Abell, and T.M. Monro, "Fluorescence-based aluminum ion sensing using a surface-functionalized microstructured optical fiber," Langmuir, 27, 5680-5685 (2011).

[24] E.P. Schartner, D. Jin, H. Ebendorff-Heidepriem, J.A. Piper, Z. Lu, and T.M. Monro, "Lanthanide up conversion within microstructured optical fibers: improved detection limits for sensing and the demonstration of a new tool for nanocrystal characterization," Nanoscale, 4, 7448-7451 (2012).

[25] S.C. Warren-Smith and T.M. Monro, "Exposed core microstructured optical fiber Bragg gratings: refractive index sensing," Opt. Express, 22, 1480-1489 (2014).

[26] S.C. Warren-Smith, R. Kostecki, L.V. Nguyen, and T.M. Monro, "Fabrication, splicing, Bragg grating writing, and polyelectrolyte functionalization of exposed-core microstructured optical fibers," Opt. Express, 22, 29493-29504 (2014).

[27] A. Francois, T. Reynolds, and T.M. Monro, "A fiber-tip label-free biological sensing platform: a practical approach toward in-vivo sensing," Sensors (Basel), 15, 1168-1181 (2015).

[28] P.St.J. Russell, "Photonic crystal fibers," Science, 299, 358-362 (2003).

[29] J.C. Knight, "Photonic crystal fibers," Nature, 424, 847-851 (2003).

[30] C.M.B. Cordeiro, M.A.R. Franco, G. Chesini, E.C.S. Barretto, R. Lwin, C.H.B. Cruz, and M.C.J. Large, "Microstructured-core optical fibre for evanescent sensing applications," Opt. Express, 14, 13056-13066 (2006).

[31] Md.I. Islam, K. Ahmed, S. Sen, S. Chowdhury, B.K. Paul, Md.S. Islam, M.B.A. Miah, and S. Asaduzzaman, "Design and optimization of photonic crystal fiber based 
sensor for gas condensate and air pollution monitoring," Photonic Sens., 7, 234-245 (2017).

[32] J.B. Jensen, P.E. Hoiby, G. Emiliyanov, O. Bang, L. Pedersen, and A. Bjarklev, "Selective detection of antibodies in microstructured polymer optical fibers," Opt. Express, 13, 5883-5889 (2005).

[33] J.M. Fini, "Microstructure fibres for optical sensing in gases and liquids," Meas. Sci. Technol., 15, 1120-1128 (2004).

[34] C.M.B. Cordeiro, E.M. dos Santos, C.H.B. Cruz, C.J. de Matos, and D.S. Ferreira, "Lateral access to the holes of photonic crystal fibers-selective filling and sensing applications," Opt. Express, 14, 8403-8412 (2006).

[35] P.S. Kumar, C.P.G. Vallabhan, V.P.N. Nampoori, V.N.S. Pillai, and P. Radhakrishnan, "A fibre optic evanescent wave sensor used for the detection of trace nitrites in water," J. Opt. A: Pure Appl. Opt., 4, 247-250 (2002).

[36] P.J. Wiejata, P.M. Shankar, and R. Mutharasan, "Fluorescent sensing using biconical tapers," Sens. Actuators B, 96, 315-320 (2003).

[37] P.J. Scully, D. Jones, and D.A. Jaroszynski, "Femtosecond laser irradiation of polymethylmethacrylate for refractive index gratings," J. Opt. A: Pure Appl. Opt., 5, S92-S96 (2003).

[38] G. Stewart, W. Jin, and B. Culshaw, "Prospects for fibre-optic evanescent-field gas sensors using absorption in the near-infrared," Sens. Actuators B Chem., 38, 42-47 (1997).

[39] Md.I. Islam, B.K. Paul, K. Ahmed, Md.R. Hasan, S. Chowdhury, Md.S. Islam, S. Sena, A.N. Bahara, and S. Asaduzzaman, "Highly birefringent single mode spiral shape photonic crystal fiber based sensor for gas sensing applications," Sensing and Bio-sensing Research, 14, 30-38 (2018).

[40] A.S.H. Rabee, Md.F.O. Hameed, A.M. Heikal, and S.S.A. Obayya, "Highly sensitive photonic crystal fiber gas sensor,” Optik, 188, 78-86 (2019).

[41] Md.F.H. Arif and Md.J.H. Biddut, "A new structure of photonic crystal fiber with high sensitivity, high nonlinearity, high birefringence and low confinement loss for liquid analyte sensing applications," Sensing and Bio-sensing Research, 12, 8-14 (2017).

[42] F.M. Cox, A. Argyros, and M.C.J. Large, "Liquid-filled hollow core microstructured polymer optical fiber," Opt. Express, 14, 4135-4140 (2006).

[43] Y.L. Hoo, W. Jin, C. Shi, H.L. Ho, D.N. Wang, and S.C. Ruan, "Design and modeling of a photonic crystal fiber gas sensor,” Appl. Opt., 42, 3509-3515 (2003). 
[44] M.A. van Eijkelenborg, M.C.J. Large, A. Argyros, J. Zagari, S. Manos, N.A. Issa, I. Bassett, S. Fleming, R.C. McPhedran, C.M.de Sterke, and N.A.P. Nicorovici, “Microstructured polymer optical fiber," Opt. Express, 9, 319-327 (2001).

[45] G. Barton, M.A. van Eijkelenborg, G. Henry, M.C.J. Large, and J. Zagari, "Fabrication of microstructured polymer optical fibers," Opt. Fiber Technol., 10, 325-335 (2004).

[46] C. Markos, W. Yuan, K. Vlachos, G.E. Town, and O. Bang, "Label-free biosensing with high sensitivity in dual-core microstructured polymer optical fibers," Opt. Express, 19, 7790-7798 (2011).

[47] C. Markos, I. Kubat, and O. Bang, "Hybrid polymer photonic crystal fiber with integrated chalcogenide glass nanofilms," Sci. Rep., 4, 6057 (2014).

[48] C.A.F. Marques, G.D. Peng, and D.J. Webb, "Highly sensitive liquid level monitoring system utilizing polymer fiber Bragg gratings,” Opt. Express, 23, 6058-6072 (2015).

[49] A.R. Prado, A.G. Leal-Junior, C. Marques, S. Leite, G.L. de Sena, L.C. Machado, A. Frizera, M.R.N. Ribeiro, and M.J. Pontes, "Polymethylmethacrylate (PMMA) recycling for the production of optical fiber sensor systems," Opt. Express, 25, 30051-30060 (2017).

[50] X. Hu, D. Saez-Rodriguez, C. Marques, O. Bang, D.J. Webb, P. Megret, and C. Caucheteur, "Polarization effects in polymer FBGs: study and use for transverse force sensing," Opt. Express, 23, 4581-4590 (2015).

[51] L. Rovati, P. Fabbri, L. Ferrari, and F. Pilati, "Plastic optical fiber pH sensor using a sol-gel sensing matrix," Fiber Opt. Sens., 5, 415-439 (2012).

[52] C. Broadway, R. Min, A.G. Leal-Junior, C. Marques, and C. Caucheteur, "Toward commercial polymer fiber Bragg grating sensors: review and applications," J. Light. Technol., 37, 2605-2615 (2019).

[53] Md.F.H. Arif, Md.M. Hossain, N. Islam, and S.M. Khaled, "A nonlinear photonic crystal fiber for liquid sensing application with high birefringence and low confinement loss," Sensing and Bio-sensing Research, 22, 100252 (2019).

[54] D.K. Sharma and S.M. Tripathi, "Analysis of the beam divergence for one-rod core microstructured optical fibres," Opto-Electronics Review, 27, 224-231 (2019).

[55] X.-F. Bao, X.-J. Wang, H. Su, and X.-J. Shu, "Geometric definition of the Vparameter in photonic crystal fibers," Opt. Lett., 39, 892-895 (2014).

[56] J.C. Knight, T.A. Birks, P.St.J. Russell, and D.M. Atkin, "All-silica single-mode optical fiber with photonic crystal cladding," Opt. Lett., 21, 1547-1549 (1996). 
[57] Y. Li, C. Wang, Y. Chen, M. Hu, B. Liu, and L. Chai, "Solution of the fundamental space-filling mode of photonic crystal fibers: numerical method versus analytical approaches,” Appl. Phys. B, 85, 597-601 (2006).

[58] A. Ghatak and S. Lokanathan, Quantum Mechanics: Theory and Applications, New Delhi, Macmillan (1999).

[59] A.W. Snyder and J.D. Love, Optical Waveguide Theory, Chapman \& Hall, London, U.K (1983).

[60] A. Sharma, "Optimal variational method for rectangular and channel waveguides," Guided Wave Optics: Selected Topics, Viva Books Private Limited, New Delhi, 233-250 (2005).

[61] A. Sharma and J.-P. Meunier, "On the scalar modal analysis of optical waveguides using approximate methods," Opt. Commun., 281, 592-599 (2007).

[62] T.P. White, R.C. McPhedran, C.M. de Sterke, L.C. Botten, and M.J. Steel, "Confinement losses in microstructured optical fibers," Opt. Lett., 26, 1660-1662 (2001).

[63] S. Olyaee, A. Naraghi, and V. Ahmadi, "High sensitivity evanescent-field gas sensor based on modified photonic crystal fiber for gas condensate and air pollution monitoring," Optik, 125, 596-600 (2014).

[64] M. Morshed, M.F.H. Arif, S. Asaduzzaman, and K. Ahmed, "Design and characterization of photonic crystal fiber for sensing applications," Eur. Sci. J., 11, 228235 (2015).

[65] J. Park, S. Lee, S. Kim, and K. Oh, "Enhancement of chemical sensing capability in a photonic crystal fiber with a hollow high index ring defect at the center," Opt. Express, 19, 1921-1929 (2011).

[66] K. Ahmeda, M. Morsheda, S. Asaduzzaman, and Md.F.H. Arif, “Optimization and enhancement of liquid analyte sensing performance based on square-cored octagonal photonic crystal fiber," Optik, 131, 687-696 (2017).

[67] S. Olyaee, M. Seifouri, A. Nikoosohbat, and M.S.E. Abadi, "Low nonlinear effects index guiding nanostructured photonic crystal fiber," Int. J. Miner. Metall., 9, 253-257 (2015).

[68] N.A. Mortensen, J.R. Folkenberg, M.D. Nielsen, and K.P. Hansen, "Modal cut off and the $V$ parameter in photonic crystal fibers," Opt. Lett., 28, 1879-1881 (2003).

[69] M. Koshiba and K. Saitoh, "Applicability of classical optical fiber theories to holey fibers," Opt. Lett., 29, 1739-1741 (2004). 
[70] D. Marcuse, "Influence of curvature on the losses of doubly clad fibers," Appl. Opt., 21, 4208-4213 (1982).

[71] L.G. Cohen, D. Marcuse, and W.L. Mammel, "Radiating leaky-mode losses in singlemode light guides with depressed-index claddings,” IEEE J. Quant. Electron., QE-18, 1467-1472 (1982).

[72] M. Koshiba and K. Saitoh, "Simple evaluation of confinement losses in holey fibers," Opt. Commun., 253, 95-98 (2005).

[73] E. Merzbacher, "The early history of quantum tunneling," Phys. Today, 55, 44-49 (2002).

[74] J.F. Algorri, D.C. Zografopoulos, A. Tapetado, D. Poudereux, and J.M. SanchezPena, "Infiltrated photonic crystal fibers for sensing applications," Sensors, 18, 4263 (2018).

[75] B. Sun, M.-Y. Chen, Y.-K. Zhang, J.-C. Yang, J.-Q. Yao, and H.-X. Cui, "Microstructured-core photonic-crystal fiber for ultra-sensitive refractive index sensing," Opt. Express, 19, 4091-4100 (2011).

[76] L. Shao, Z. Liu, J. Hu, D. Gunawardena, and H.-Y. Tam, "Optofluidics in microstructured optical fibers," Micromachines, 9, 145 (2018).

[77] Md.F.H. Arif, K. Ahmed, S. Asaduzzaman, and Md.A.K. Azad, "Design and optimization of photonic crystal fiber for liquid sensing applications," Photonic Sens., 6, 279-288 (2016).

[78] H. Ademgil, "Highly sensitive octagonal photonic crystal fiber based sensor," Optik, $125,6274-6278$ (2014).

[79] K. Ahmed and M. Morshed, "Design and numerical analysis of microstructured-core octagonal photonic crystal fiber for sensing applications," Sensing and Bio-Sensing Research, 7, 1-6 (2016).

[80] J. Sultana, Md.S. Islam, C.M.B. Cordeiro, A. Dinovitser, M. Kaushik, B.W.-H. Ng, and D. Abbott, "Terahertz hollow core antiresonant fiber with metamaterial cladding," Fibers, 8, 14 (2020).

[81] Z. Li, H.Y. Tam, L. Xu, and Q. Zhang, "Fabrication of long-period gratings in poly (methyl methacrylateco-methyl vinyl ketone-co-benzyl methacrylate)-core polymer optical fiber by use of a mercury lamp," Opt. Lett., 30, 1117-1119 (2005).

[82] E.P. Schartner, A. Dowler, and H. Ebendorff-Heidepriem, "Fabrication of low-loss, small-core exposed core microstructured optical fibers," Opt. Mater. Express, 7, 1496$1502(2017)$. 
[83] Y. Huang, Y. Xu, and A. Yariv, "Fabrication of functional microstructured optical fibers through a selective-filling technique,” Appl. Phys. Lett., 85, 5182-5184 (2004).

[84] G.S. Kliros and P.C. Divari, "Coupling characteristics of laser diodes to high numerical aperture thermally expanded core fibers," J. Mater. Sci. Mater. Electron., 20, S59-S62, (2009).

[85] G.S. Kliros and G.K. Paschalidis, "Calculation of coupling losses between laser diodes and large mode area photonic crystal fibers," International Workshop on Fiber Optics in Acess Network (FOAN), 1-6 (2016).

[86] G.S. Kliros, “Analysis of coupling loss between laser diodes and solid-core Bragg fibers,” J. Infrared Milli. Terahz. Waves, 31, 78-87 (2010).

[87] A. Ghatak and K. Thyagarajan, Introduction to Fiber Optics, Cambridge University Press, Cambridge (1998).

[88] K. Thyagarajan and A. Ghatak, LASERS: Fundamentals and Applications, Macmillan India, New Delhi (1981).

[89] A. Ghatak and K. Thyagarajan, Optical Electronics, Cambridge University Press, Cambridge (1989).

[90] T.M. Monro, W. Belardi, K. Furusawa, J.C. Baggett, N.G.R. Broderick, and D.J. Richardson, "Sensing with microstructured optical fibers," Meas. Sci. Technol., 12, 854$858(2001)$.

[91] M. Morshed, Md.I. Hassan, T.K. Roy, Md.S. Uddin, and S.M.A. Razzak,“ Microstructure core photonic crystal fiber for gas sensing applications," Appl. Opt., 54, 8637-8643 (2015).

[92] H.C. Nguyen, B.T. Kuhlmey, E.C. Magi, M.J. Steel, P. Domachuk, C.L. Smith, and B.J. Eggleton, "Tapered photonic crystal fibres: properties, characterization and applications,” Appl. Phys. B, 81, 377-387 (2005).

[93] J. Ma, H.H. Yu, X. Jiang, and D.S. Jiang, "High-performance temperature sensing using a selectively filled solid-core photonic crystal fiber with a central air-bore," Opt. Express, 25, 9406-9415 (2017).

[94] D. Hasan and M.S. Alam, "Ultra-broadband confinement in deep sub-wavelength air hole of a suspended core fiber," J. Light. Technol., 32, 1434-1441 (2014). 
Figures
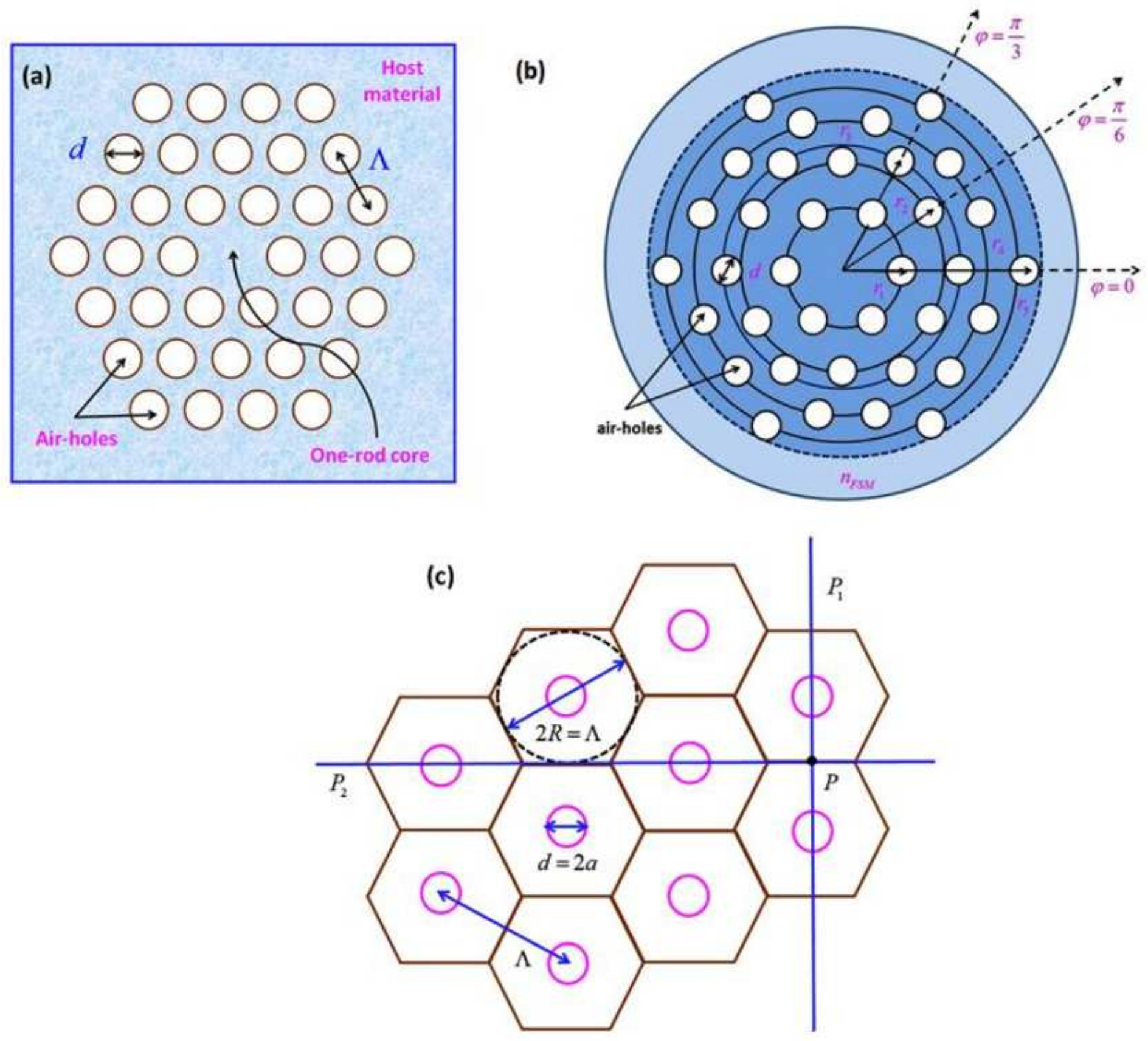

Figure 1

(a) Schematic representation of the one-rod core MOF (b) simplified sketch of the proposed model, and (c) schematic diagram for cladding structure with hexagonal and commensurate circular unit cell. 
(a)

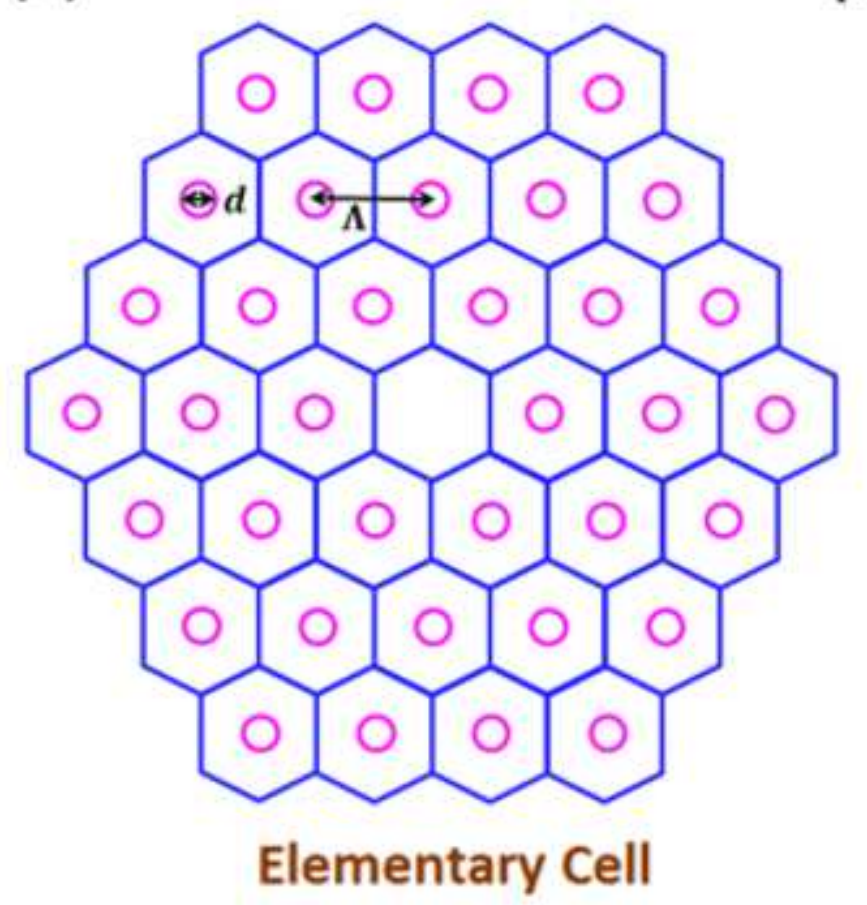

(b)

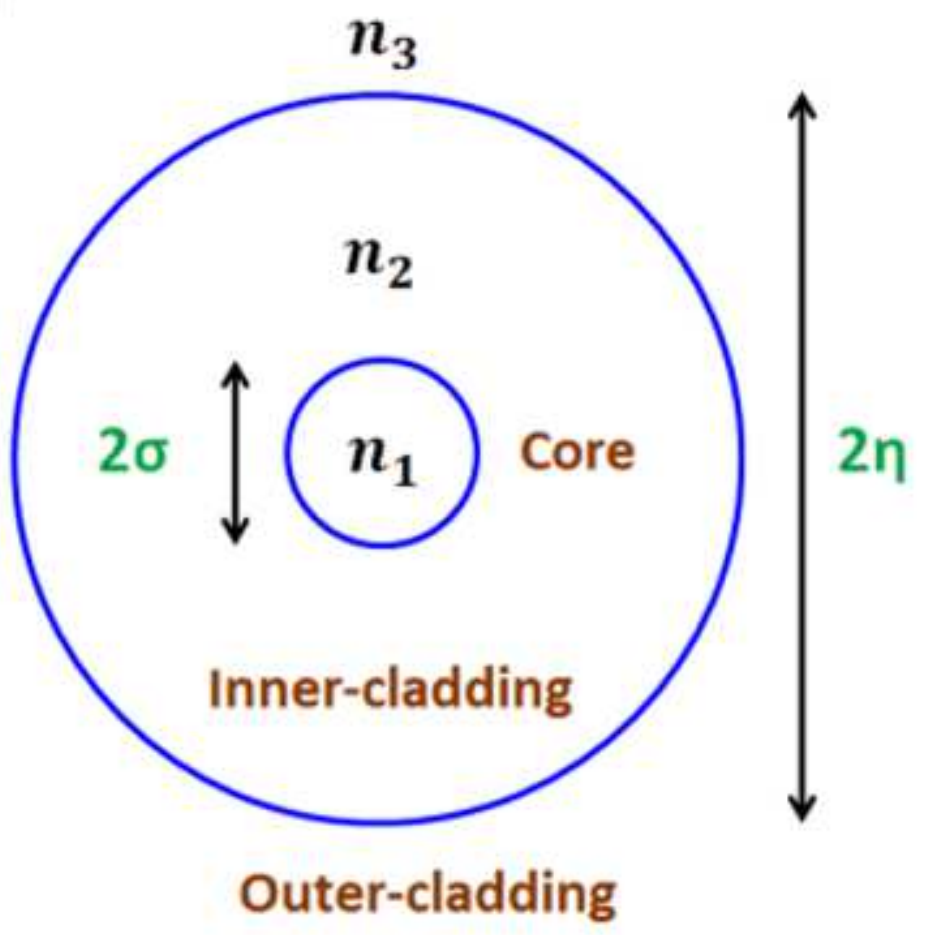

Figure 2

(a) Schematic sketch for the cross-section of solid-core MOF with finite layers of rounded air-holes (b) corresponding double-clad fiber (DCF) with a depressed index inner cladding. 

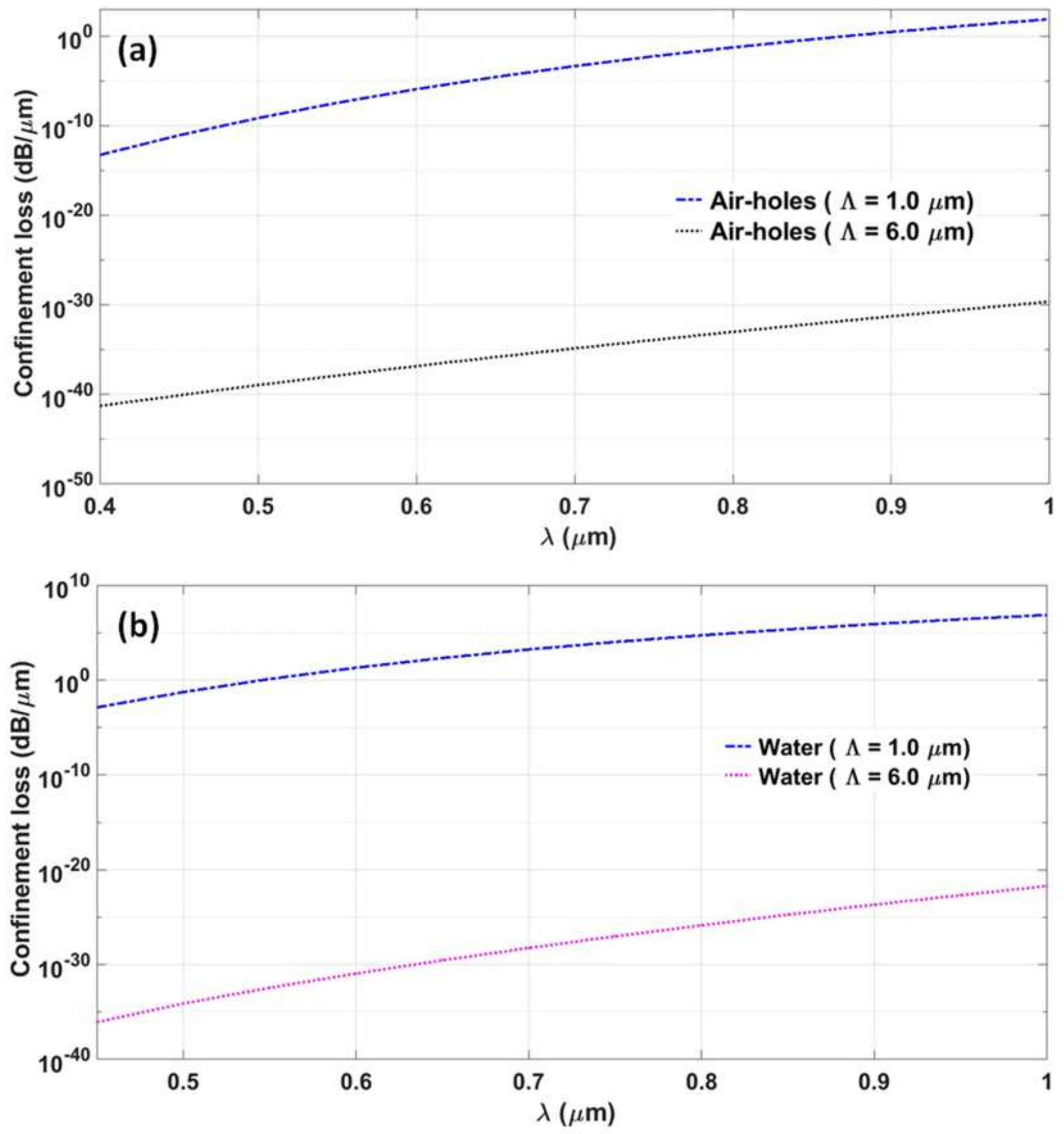

Figure 3

Spectral dependence of confinement loss for (a) Air-filled and (b) Water-filled configuration of the fibers. 


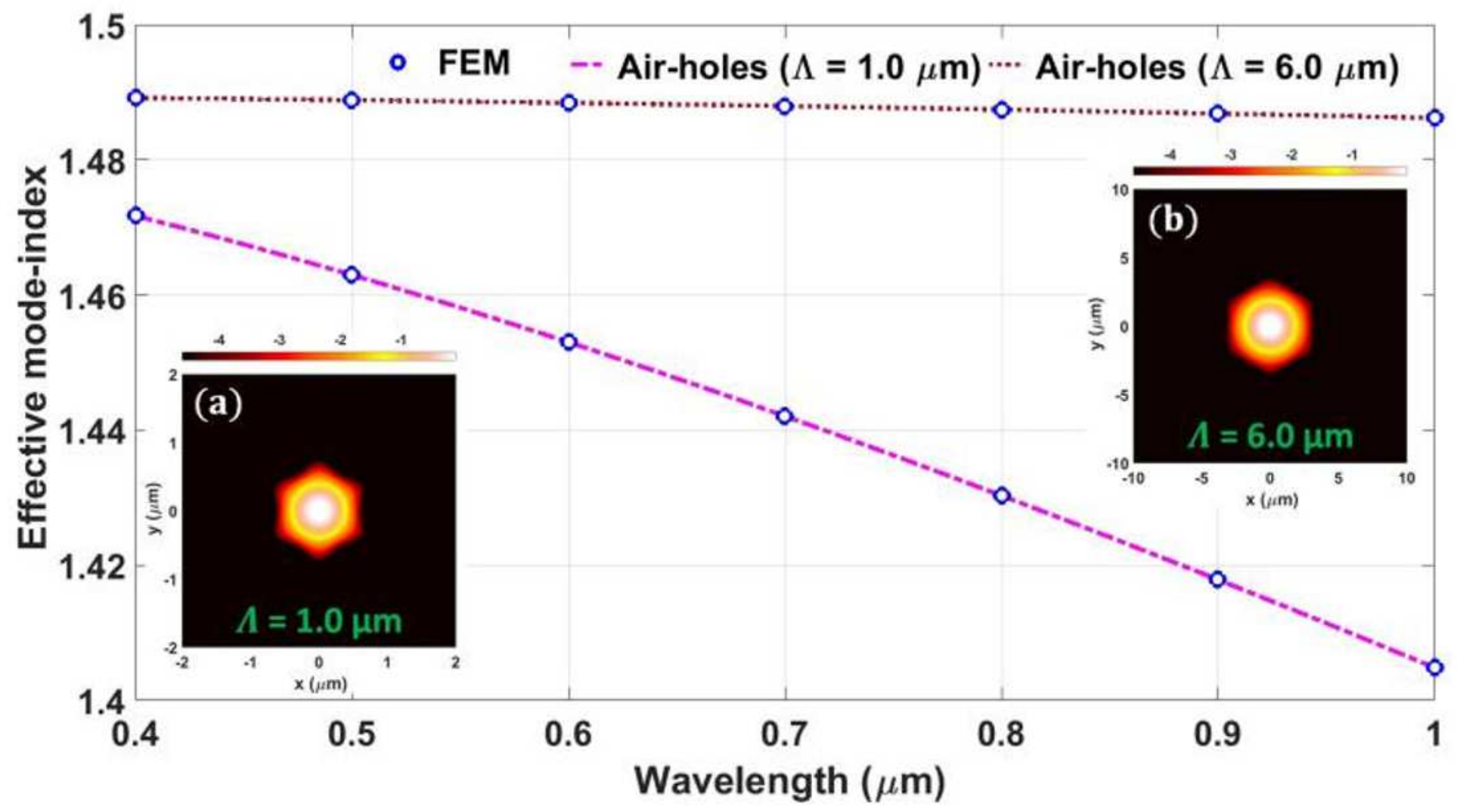

Figure 4

Spectral dependence of the effective mode-index against wavelength for the AF-configuration. The inset demonstrates their near-field intensity pattern on the logarithm scale.

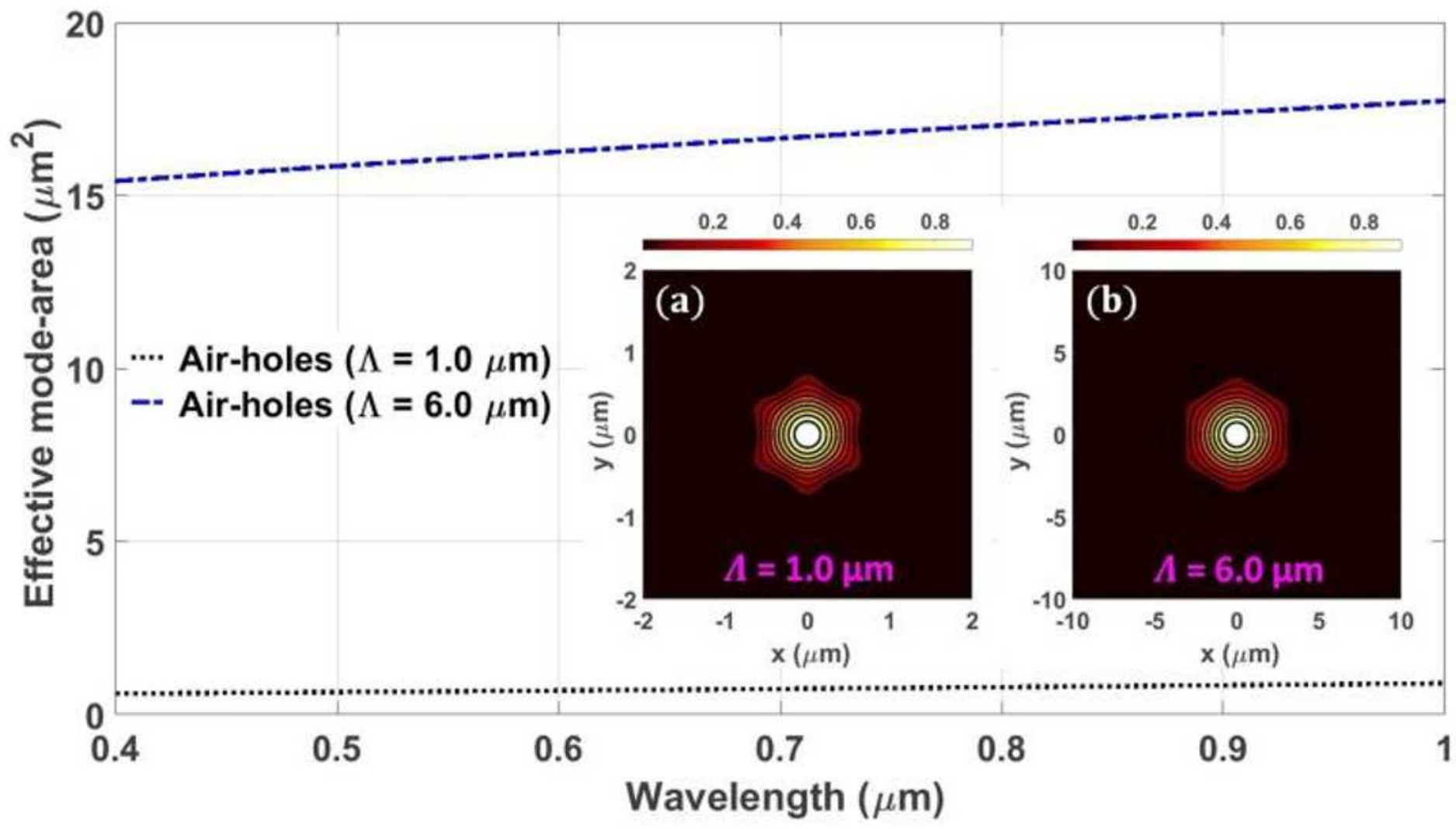


Figure 5

Effective mode-area against wavelength for AF-configuration of the introduced P-MOFs and the inset depicts their 2D surface plot for intensity distribution.

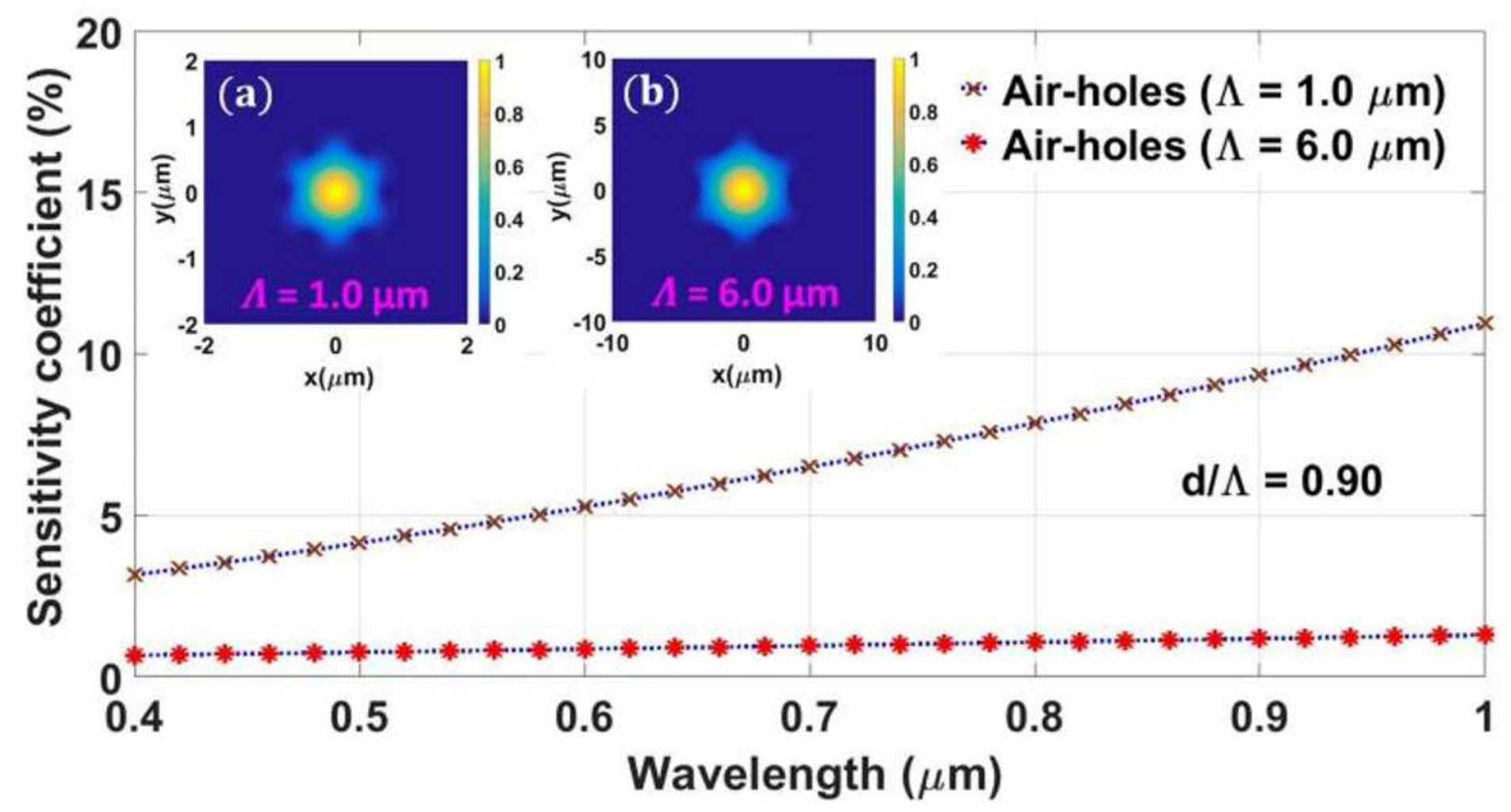

Figure 6

Sensitivity coefficient against wavelength for two different cladding configurations of P-MOFs. Inset depicts near-field of cardinal core-mode at $\Lambda=1.0 \mu \mathrm{m}$ and $6.0 \mu \mathrm{m}$, when holes are assumed to be infiltrated with air. 


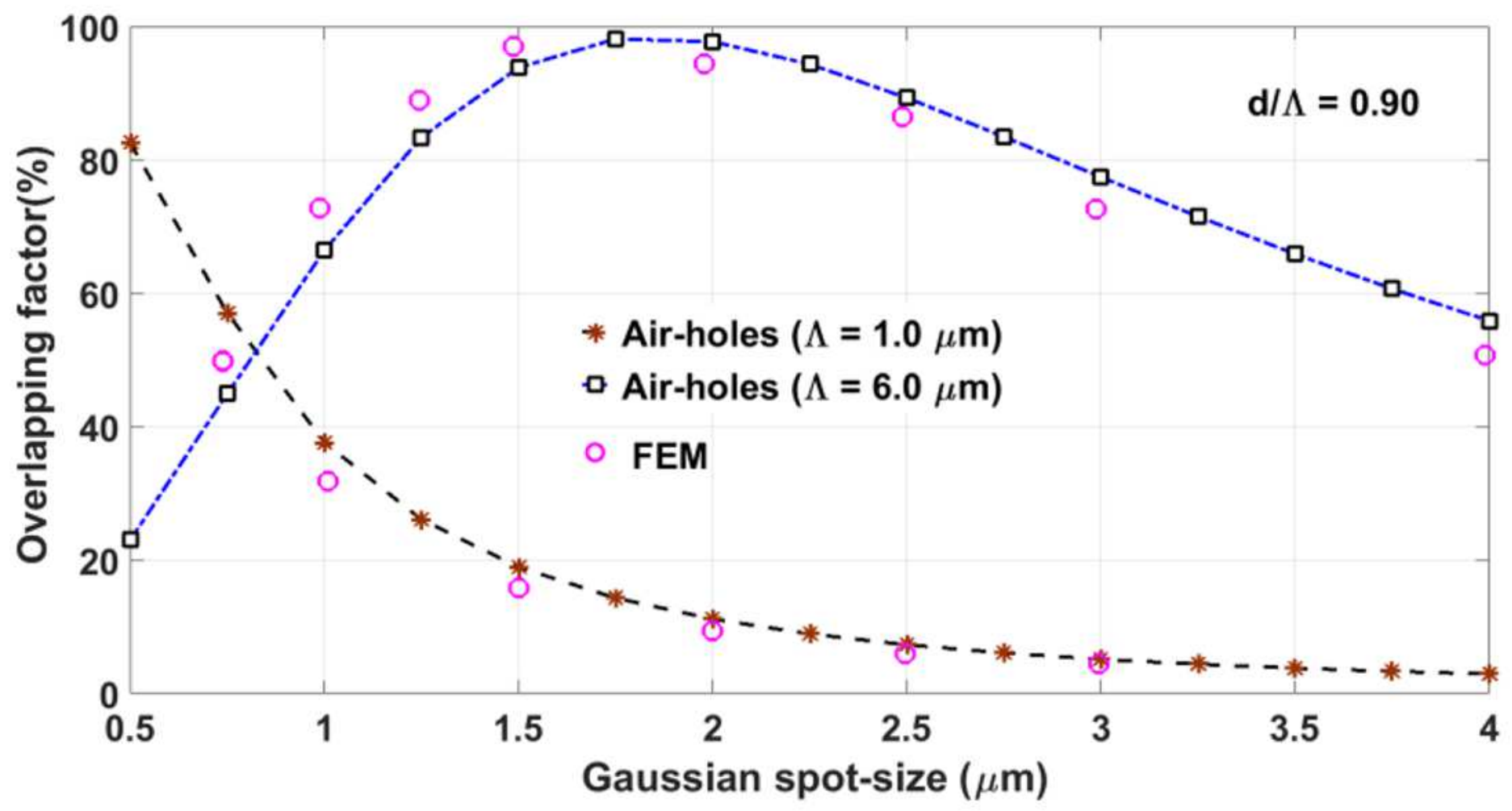

Figure 7

. Effective mode-index against wavelength for WF-configuration of the fibers. Inset illustrates their nearfield intensity pattern on a logarithm scale.

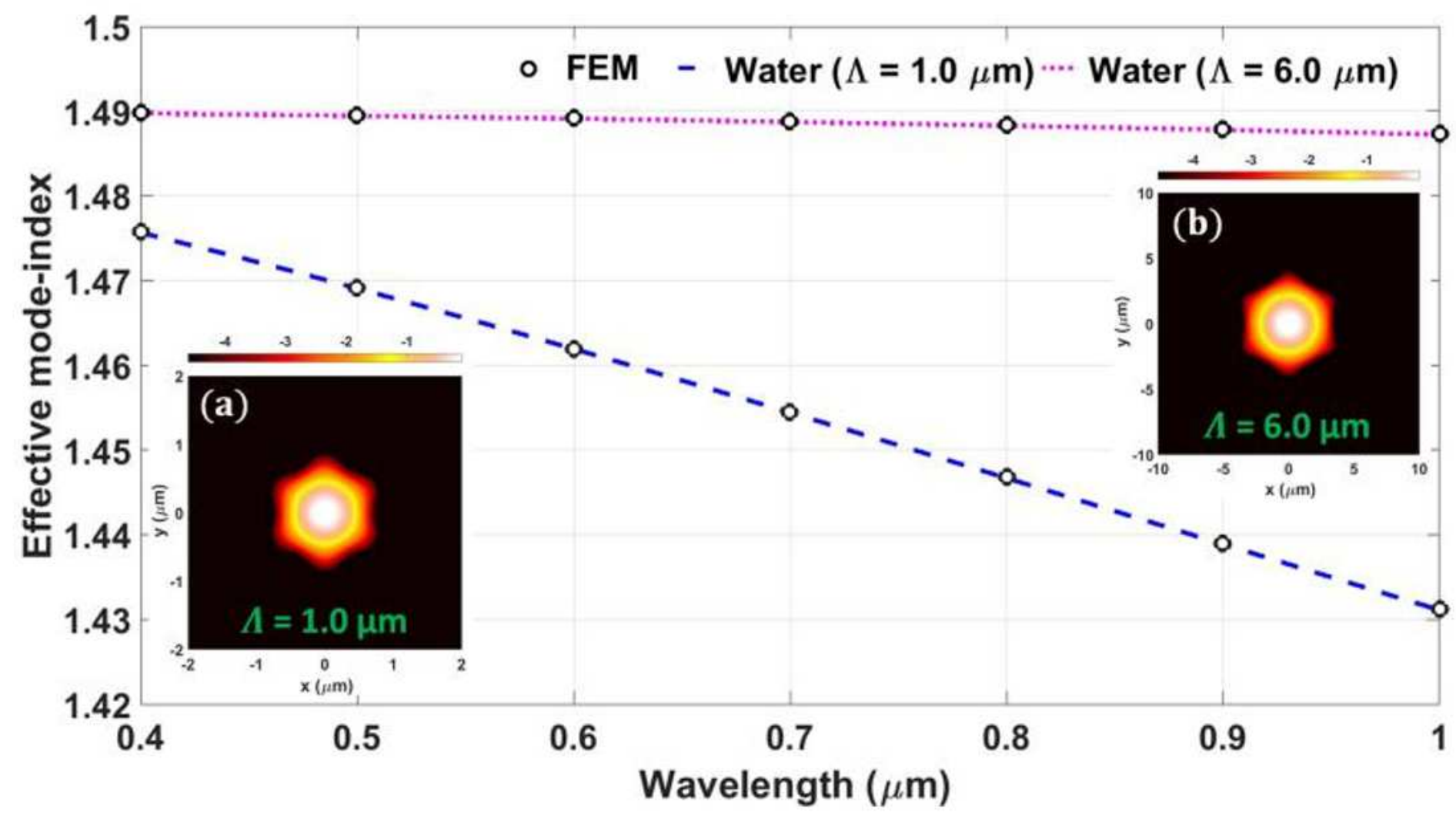


Figure 8

Effective mode-index against wavelength for WF-configuration of the fibers. Inset illustrates their nearfield intensity pattern on a logarithm scale.

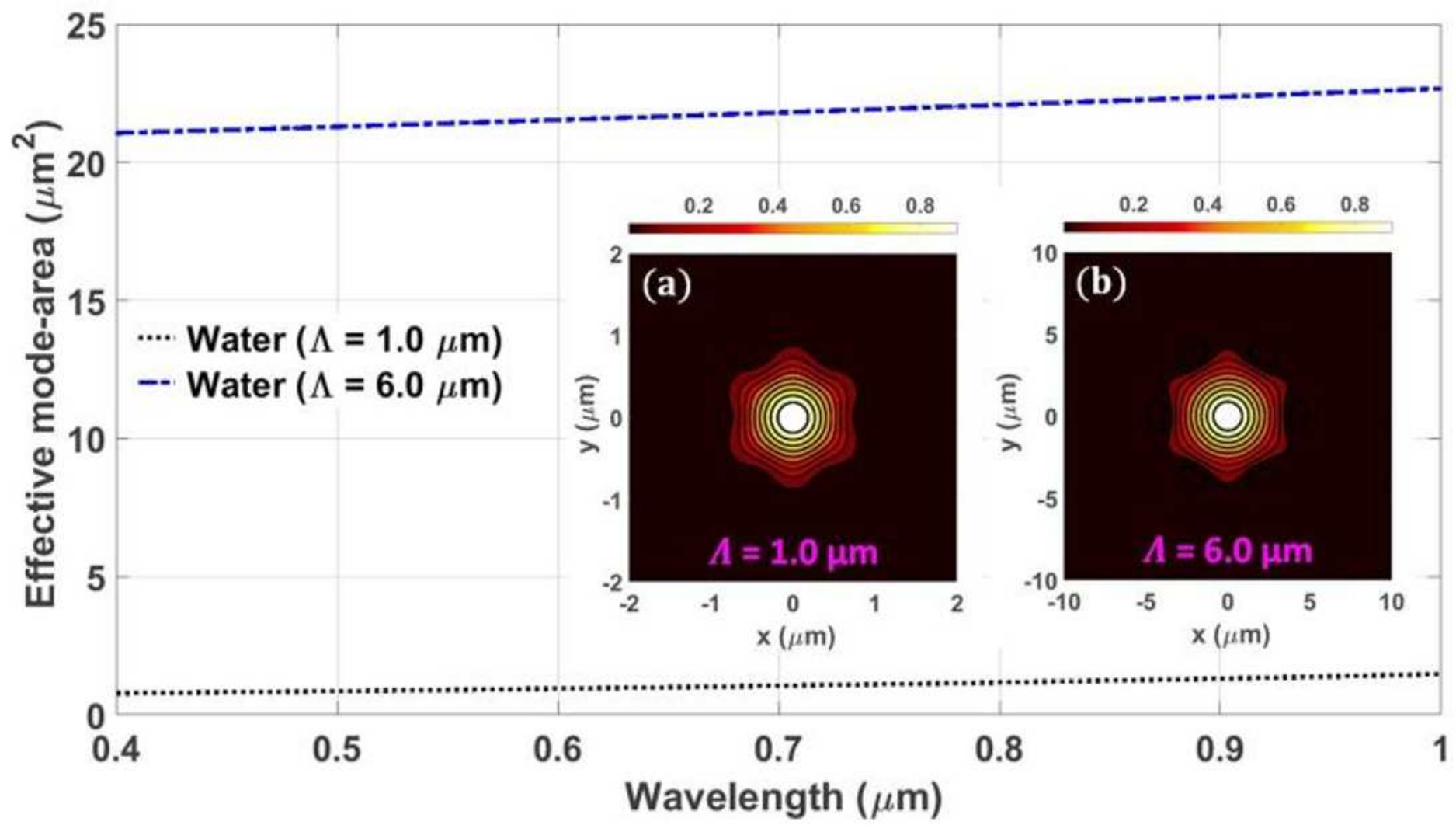

Figure 9

Effective mode-area as a function of wavelength for water-filled configuration of the introduced P-MOFs. 


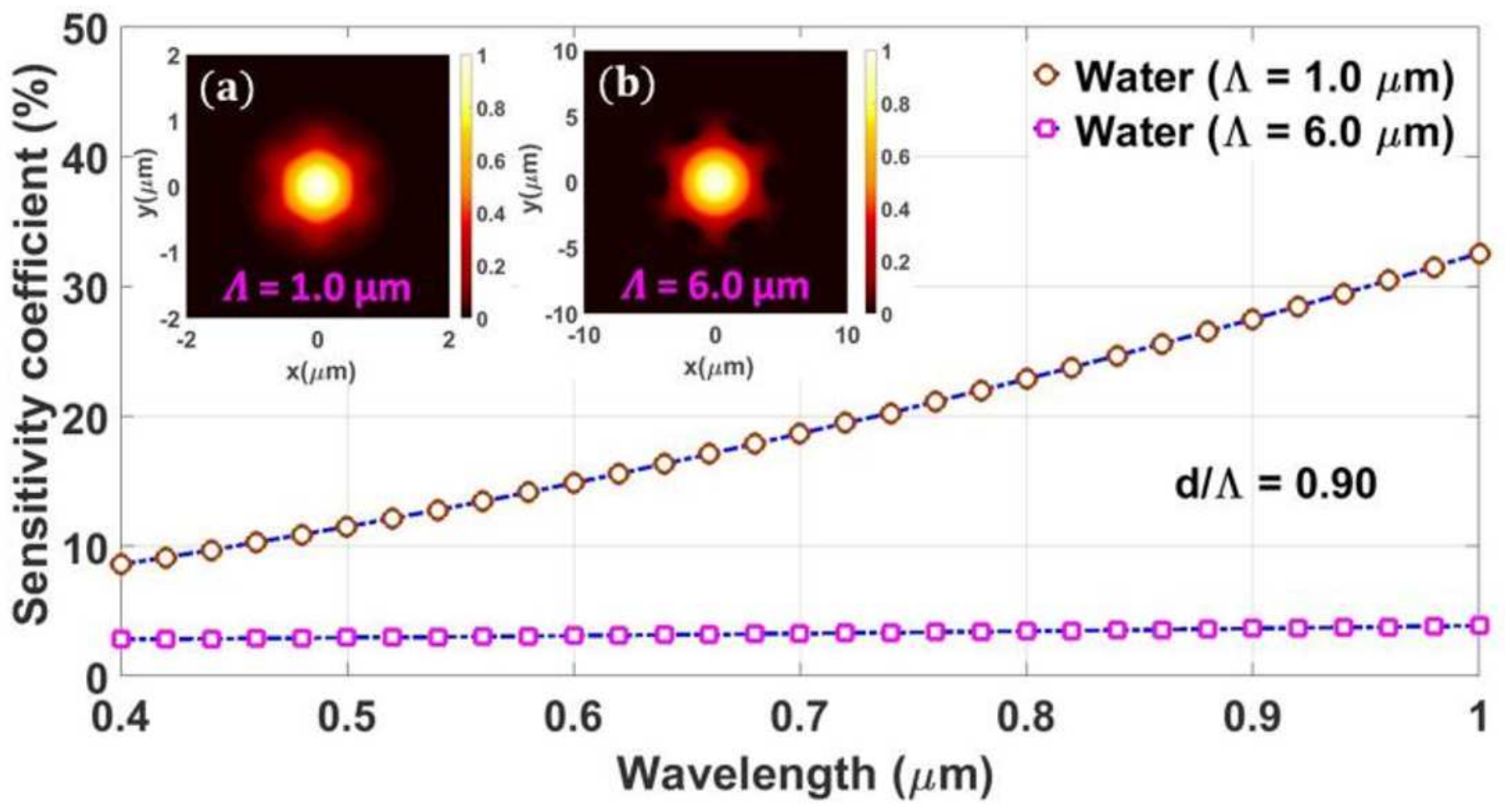

Figure 10

Sensitivity coefficient against wavelength for WF-layout. Inset depicts their near-field patterns at 0.633 $\mu \mathrm{m}$.

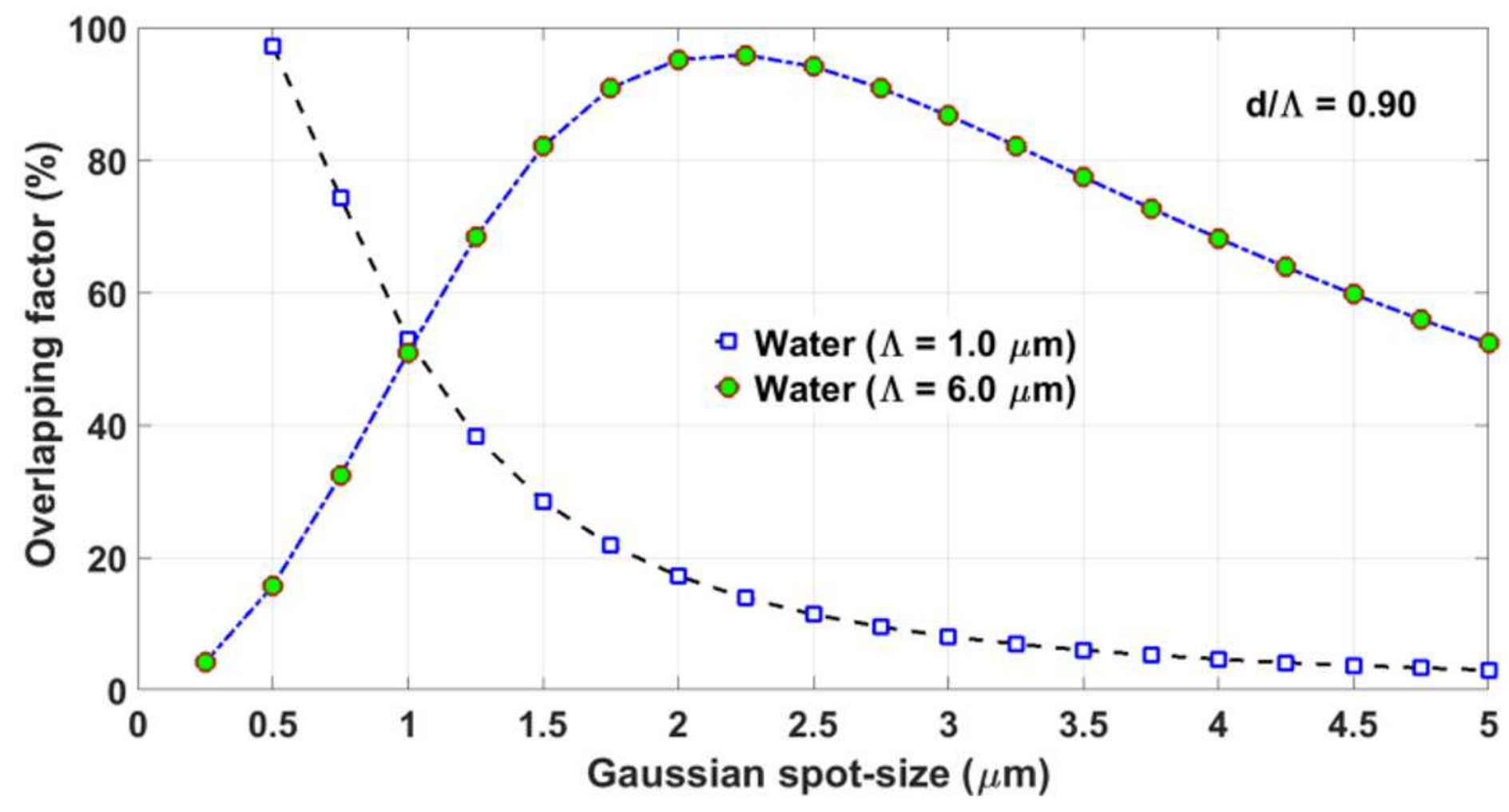

Figure 11 
Coupling efficiency against Gaussian radius between Gaussian optical mode and the core-mode of PMOF's water-filled design at $0.633 \mu \mathrm{m}$. 